\title{
BOUNDARY INTEGRAL OPERATOR FOR THE FRACTIONAL LAPLACIAN ON THE BOUNDARY OF A BOUNDED SMOOTH DOMAIN
}

\author{
TONGKEUN CHANG
}

Communicated by William McLean

\begin{abstract}
We introduce the boundary integral operator induced from the fractional Laplace equation on the boundary of a bounded smooth domain. For $\frac{1}{2}<\alpha<1$, we show the bijectivity of the boundary integral operator $S_{2 \alpha}: L^{p}(\partial \Omega) \rightarrow H_{p}^{2 \alpha-1}(\partial \Omega)$ for $1<p<\infty$. As an application, we demonstrate the existence of the solution of the Dirichlet boundary value problem of the fractional Laplace equation.
\end{abstract}

1. Introduction. In this paper, we study a boundary integral operator defined on the boundary of a smooth, bounded domain $\Omega$ in $\mathbb{R}^{n}$ for $n \geq 3$. Let $\Gamma_{2 \alpha}(x):=c(n, 2 \alpha) /|x|^{n-2 \alpha}$ be the Riesz kernel of order $2 \alpha$ in $\mathbb{R}^{n}$, where $0<2 \alpha<n$ and $c(n, 2 \alpha)$ is the usual normalization constant. The single layer potential of a fractional Laplacian for a function $\phi$, defined on $\partial \Omega$, is defined by

$$
\mathcal{S}_{2 \alpha} \phi(x):=\int_{\partial \Omega} \Gamma_{2 \alpha}(x-Q) \phi(Q) d Q, \quad x \in \mathbb{R}^{n} .
$$

Note that, if $1<2 \alpha<n$ and $\phi \in L^{\infty}(\partial \Omega)$, then $\mathcal{S}_{2 \alpha} \phi$ is continuous on $\mathbb{R}^{n}$, and we define the boundary integral operator

$$
S_{2 \alpha} \phi(P):=\int_{\partial \Omega} \Gamma_{2 \alpha}(P-Q) \phi(Q) d Q, \quad P \in \partial \Omega,
$$

by restriction of $\mathcal{S}_{2 \alpha} \phi$ to $\partial \Omega$.

2010 AMS Mathematics subject classification. Primary 30E25, 45P05.

Keywords and phrases. Boundary integral operator, layer potential, fractional Laplacian.

The author was supported by grant No. NRF20151009350.

Received by the editors on September 22, 2015, and in revised form on April 29, 2016. 
Zähle [22, 23] studied the Riesz potentials in a general metric space $(X, \rho)$ with Ahlfors $d$-regular measure $\mu$. She demonstrated that $S_{2 \alpha}: L^{2}(X, d \mu) \rightarrow L_{2 \alpha}^{2}(X, d \mu)$ is invertible for $0<2 \alpha<n$, where $L^{2}(X, d \mu)$ is decomposed by the null space, $N\left(S_{2 \alpha}\right)$, and its orthogonal compliment, that is, $L^{2}(X, d \mu)=N\left(S_{2 \alpha}\right) \otimes L_{2 \alpha}^{2}(X, d \mu)$.

The eigenvalue asymptotic behavior for integral operators of potential types on a Lipschitz surface was studied by Agranovich and Amosov [1] and by Rozenblum and Tashchiyan [17]. Chang [6] showed that the boundary integral operator $S_{2 \alpha}$ defined in (1.2) extends to a bijective operator $S_{2 \alpha}: H_{2}^{-\alpha+1 / 2}(\partial \Omega) \rightarrow H_{2}^{\alpha-1 / 2}(\partial \Omega)$ for $1 / 2<\alpha<1$, and that $\mathcal{S}_{2 \alpha} \phi \in \dot{H}_{2}^{\alpha}\left(\mathbb{R}^{n}\right)$ for $\phi \in H_{2}^{-\alpha+1 / 2}(\partial \Omega)$; see Section 2 for the definitions of function spaces.

When $2 \alpha=2, \Gamma_{2}$ is the fundamental solution of the Laplace equation in $\mathbb{R}^{n}$, and (1.1 is the single layer potential of the Laplace equation. The single layer potential and boundary layer potential of the Laplace equation have been studied by many mathematicians to demonstrate the existence of a solution to a boundary value problem of the Laplace equation in a bounded domain $[\mathbf{9}, \mathbf{1 1}, \mathbf{1 4}, \mathbf{2 1}]$.

The first result of this paper is the following theorem. The function space $H_{p}^{s}(\partial \Omega)$ is defined in Section 2 .

Theorem 1.1. Let $\Omega$ be a bounded $C^{2}$-domain in $\mathbb{R}^{n}$ with $n \geq 3$. Let $1 / 2<\alpha<1$ and $1<p<\infty$. Then, $S_{2 \alpha}: L^{p}(\partial \Omega) \rightarrow H_{p}^{2 \alpha-1}(\partial \Omega)$ is bijective.

The layer potential for $\phi \in B_{p}^{s}(\partial \Omega)$, for $s<0$, and $1<p<\infty$ is defined by

$$
\mathcal{S}_{2 \alpha} \phi(x)=\left\langle\phi, \Gamma_{2 \alpha}(x-\cdot)\right\rangle, \quad x \in \mathbb{R} \backslash \partial \Omega,
$$

where $\langle\cdot, \cdot\rangle$ is the duality pairing between $B_{p}^{s}(\partial \Omega)$ and $B_{p^{\prime}}^{-s}(\partial \Omega)$, for $1 / p+1 / p^{\prime}=1$. In particular, if $\phi \in L^{p}(\partial \Omega)$, then $\mathcal{S}_{2 \alpha} \phi$ is defined by (1.1). The second result is the following theorem. The function spaces $B_{\text {loc }, p}^{s}\left(\mathbb{R}^{n}\right)$ and $\dot{B}_{p}^{s}\left(\mathbb{R}^{n}\right)$ are defined in Section 2 .

Theorem 1.2. Let $1 / 2<\alpha<1$ and $1<p<\infty$. For $\phi \in B_{p}^{s}(\partial \Omega)$, let $u=\mathcal{S}_{2 \alpha} \phi$ be the layer potential defined in (1.3). Let $-2 \alpha+1-1 / p<$ 
$s<0$. Then $u \in B_{\mathrm{loc}, p}^{s+2 \alpha-1+1 / p}\left(\mathbb{R}^{n}\right)$, and

$$
\|u\|_{B_{p}^{s+2 \alpha-1+1 / p}\left(B_{R}\right)} \leq c_{R}\|\phi\|_{B_{p}^{s}(\partial \Omega)}
$$

where $B_{R}$ denotes the open ball in $\mathbb{R}^{n}$ whose radius is $R$ and whose center is the origin, and $R$ is chosen sufficiently large that $\Omega \subset B_{R}$. Moreover, if $p>(n-1) /(n+s-1)$, then $u \in \dot{B}_{p}^{s+2 \alpha-1+1 / p}\left(\mathbb{R}^{n}\right)$, and

$$
\|u\|_{\dot{B}_{p}^{s+2 \alpha-1+1 / p}\left(\mathbb{R}^{n}\right)} \leq c\|\phi\|_{B_{p}^{s}(\partial \Omega)} .
$$

Boundary integral operators such as the single and double layer potentials have been studied by many mathematicians. The bijectivity of these operators has been used to demonstrate the existence of the solutions to partial differential equations in a bounded domain or bounded cylinder $[4,5,8, \mathbf{1 0}, \mathbf{1 3}, \mathbf{1 6}, \mathbf{1 8}]$. Extending this approach, we apply the bijectivity of the boundary integral operator to the boundary value problem of the fractional Laplace equation. The fractional Laplacian of order $0<\alpha<1$ of a function $v: \mathbb{R}^{n} \rightarrow \mathbb{R}$ may be defined by the formula:

$$
(-\triangle)^{\alpha} v(x):=C(n, \alpha) \int_{\mathbb{R}^{n}} \frac{-v(x+y)+2 v(x)-v(x-y)}{|y|^{n+2 \alpha}} d y
$$

where $C(n, \alpha)$ is a normalization constant. The fractional Laplacian can also be defined as a pseudo-differential operator,

$$
\widehat{(-\triangle)^{\alpha}} v(\xi)=(2 \pi|\xi|)^{2 \alpha} \widehat{v}(\xi),
$$

where $\widehat{v}(\xi):=\int_{\mathbb{R}^{n}} v(x) e^{-2 \pi i \xi \cdot x} d x, \xi \in \mathbb{R}^{n}$, is the Fourier transform of $v$ in $\mathbb{R}^{n}$. In particular, when $2 \alpha=2$, the classical Laplacian is

$$
\triangle v(x):=\sum_{1 \leq i \leq n} \partial^{2} v / \partial x_{i}^{2}
$$

Definition 1.3. Let $0<\alpha<1$. We say that $v$ is a weak solution of $(-\triangle)^{\alpha} u=0$ in $\mathbb{R}^{n} \backslash \partial \Omega$ if $v$ satisfies, for all $\psi \in C_{\mathrm{c}}^{\infty}\left(\mathbb{R}^{n} \backslash \partial \Omega\right)$,

$$
\int_{\mathbb{R}^{n}} v(x)(-\triangle)^{\alpha} \psi(x) d x=\int_{\mathbb{R}^{n}}(2 \pi|\xi|)^{2 \alpha} \widehat{v}(\xi) \bar{\psi}(\xi) d \xi=0 .
$$


In fact, if $u$ is a weak solution, then $u$ is a continuous function in $\mathbb{R} \backslash \partial \Omega$ and satisfies [3, Theorem 3.9]

$$
(-\triangle)^{\alpha} u(x)=0 \quad \text { for } x \in \mathbb{R}^{n} \backslash \partial \Omega \text {. }
$$

For the application of Theorem 1.1 and Theorem 1.2, we show the existence of a solution to the boundary value problem of the fractional Laplace equation.

Theorem 1.4. Let $\Omega$ be a bounded $C^{2}$ domain in $\mathbb{R}^{n}$, for $n \geq 3$, and let $1 / 2<\alpha<1,0<t<2 \alpha-1$ and $1<p<\infty$. Then, for given $g \in B_{p}^{t}(\partial \Omega)$, the boundary value problem

$$
\begin{gathered}
(-\triangle)^{\alpha} u=0 \quad \text { in } \mathbb{R}^{n} \backslash \partial \Omega,\left.\quad u\right|_{\partial \Omega}=g \quad \text { on } B_{p}^{t}(\partial \Omega), \\
|u(x)|=O\left(|x|^{-n+2 \alpha}\right) \quad \text { as }|x| \rightarrow \infty,
\end{gathered}
$$

has a weak solution $u \in B_{\mathrm{loc}, p}^{t+1 / p}\left(\mathbb{R}^{n}\right)$. In addition, $u \in \dot{B}_{p}^{t+1 / p}\left(\mathbb{R}^{n}\right)$ if $(n-1) /(n+t-2 \alpha)<p<\infty$, and there exists $\phi \in B_{p}^{t-2 \alpha+1}(\partial \Omega)$ such that

$$
u=\mathcal{S}_{2 \alpha} \phi
$$

The rest of this paper is organized as follows. In Section 2, we introduce several function spaces, and in Section 3, we introduce several properties of the layer potential. In Sections 4,5 and 6 , we prove Theorems 1.1, 1.2 and 1.4, respectively.

\section{Function spaces.}

2.1. Function spaces in $\mathbb{R}^{n}$. In this section, we introduce Sobolev and Besov spaces. For $s \in \mathbb{R}$, we consider a distribution $G_{s}$, whose Fourier transform in $\mathbb{R}^{n}$ is defined by

$$
\widehat{G_{s}}(\xi)=\left(1+4 \pi^{2}|\xi|^{2}\right)^{-s / 2} .
$$

For $s \in \mathbb{R}$ and $1 \leq p \leq \infty$, we define the Sobolev space $H_{p}^{s}\left(\mathbb{R}^{n}\right)$ by

$$
H_{p}^{s}\left(\mathbb{R}^{n}\right):=\left\{f \in \mathcal{S}^{\prime}\left(\mathbb{R}^{n}\right):\|f\|_{H_{p}^{s}(\mathbb{R})}:=\left\|G_{-s} * f\right\|_{L^{p}\left(\mathbb{R}^{n}\right)}<\infty\right\},
$$

where $*$ is the usual Fourier convolution in $\mathbb{R}^{n}$ and $\mathcal{S}^{\prime}\left(\mathbb{R}^{n}\right)$ is the dual space of the Schwartz space $\mathcal{S}\left(\mathbb{R}^{n}\right)$. In particular, when $s=k \in$ 
$\mathbb{N} \cup\{0\}$ and $1<p<\infty$,

$$
H_{p}^{k}\left(\mathbb{R}^{n}\right)=\left\{f: D^{\beta} f \in L^{p}\left(\mathbb{R}^{n}\right) \text { for }|\beta| \leq k\right\},
$$

where $\beta=\left(\beta_{1}, \beta_{2}, \ldots, \beta_{n}\right) \in(\mathbb{N} \cup\{0\})^{n}$ and $|\beta|:=\beta_{1}+\beta_{2}+\cdots+\beta_{n}$.

For $k<s<k+1$ and $k \in \mathbb{N}$, we define the seminorm,

$$
|f|_{B_{p}^{s}}:=\left(\sum_{|\beta|=k} \iint_{\mathbb{R}^{n} \times \mathbb{R}^{n}} \frac{\left|D^{\beta} f(x)-D^{\beta} f(y)\right|^{p}}{|x-y|^{n+p(s-k)}} d y d x\right)^{1 / p},
$$

and note that $|f+g|_{B_{p}^{s}\left(\mathbb{R}^{n}\right)}=|f|_{B_{p}^{s}\left(\mathbb{R}^{n}\right)}$ if $g$ belongs to the space $\mathbb{P}_{k}\left(\mathbb{R}^{n}\right)$ of polynomials of degree $k$ or less on $\mathbb{R}$. Then,

$$
B_{p}^{s}\left(\mathbb{R}^{n}\right):=\left\{f \in \mathcal{S}^{\prime}\left(\mathbb{R}^{n}\right):\|f\|_{B_{p}^{s}}<\infty\right\},
$$

with the norm $\|f\|_{B_{p}^{s}}:=\|f\|_{H_{p}^{k}}+|f|_{B_{p}^{s}}$, and $\left\|f+\mathbb{P}_{k}\left(\mathbb{R}^{n}\right)\right\|_{\dot{B}_{p}^{s}\left(\mathbb{R}^{n}\right)}:=$ $|f|_{B_{p}^{s}\left(\mathbb{R}^{n}\right)}$ is an equivalent norm on the quotient space $\dot{B}_{p}^{s}\left(\mathbb{R}^{n}\right):=$ $B_{p}^{s}\left(\mathbb{R}^{n}\right) / \mathbb{P}_{k}\left(\mathbb{R}^{n}\right)$. If $s \in \mathbb{R}$ is negative, then we define $B_{p}^{s}$ and $\dot{B}_{p}^{s}$ as the dual spaces of $B_{p^{\prime}}^{-s}$ and $\dot{B}_{p^{\prime}}^{-s}$, respectively, where $1 / p+1 / p^{\prime}=1$. The real and complex interpolation methods [2, Theorem 6.4.5] give

$$
\left(H_{p}^{s_{0}}, H_{p}^{s_{1}}\right)_{\theta, p}=B_{p}^{s} \quad \text { and } \quad\left[H_{p}^{s_{0}}, H_{p}^{s_{1}}\right]_{\theta}=H_{p}^{s}
$$

for $s=(1-\theta) s_{0}+\theta s_{1}, s_{0}, s_{1} \in \mathbb{R}$ and $0<\theta<1$.

2.2. Function spaces in $\Omega$. Let $\Omega$ be a bounded $C^{2}$-domain in $\mathbb{R}^{n}$, and, for a function $f$ defined on $\mathbb{R}^{n}$, let $R_{\Omega} f$ denote the restriction of $f$ to $\Omega$. For $s \geq 0$, we define the function spaces

$$
H_{p}^{s}(\Omega):=\left\{R_{\Omega} f: f \in H_{p}^{s}\left(\mathbb{R}^{n}\right)\right\}
$$

and

$$
B_{p}^{s}(\Omega):=\left\{R_{\Omega} f: f \in B_{p}^{s}\left(\mathbb{R}^{n}\right)\right\}
$$

with norms

$$
\|f\|_{H_{p}^{s}(\Omega)}:=\inf \|F\|_{H_{p}^{s}\left(\mathbb{R}^{n}\right)}
$$

and

$$
\|f\|_{B_{p}^{s}(\Omega)}:=\inf \|F\|_{B_{p}^{s}\left(\mathbb{R}^{n}\right)},
$$


where infimums are taken over all $F$ in $H_{p}^{s}\left(\mathbb{R}^{n}\right)$ and $F \in B_{p}^{s}\left(\mathbb{R}^{n}\right)$, respectively, such that $R_{\Omega} F=f$.

Note that, for a non-negative integer $k$ and for $1<p<\infty$,

$$
H_{p}^{k}(\Omega)=\left\{f \in L^{p}(\Omega): D^{\beta} f \in L^{p}(\Omega) \quad \text { for }|\beta| \leq k\right\}
$$

and, for $0<\theta<1$,

$$
\left(H_{p}^{k_{0}}(\Omega), H_{p}^{k_{1}}(\Omega)\right)_{\theta, p}=B_{p}^{s}(\Omega)
$$

and

$$
\left[H_{p}^{k_{0}}(\Omega), H_{p}^{k_{1}}(\Omega)\right]_{\theta}=H_{p}^{s}(\Omega),
$$

where $s=(1-\theta) k_{0}+\theta k_{1}$; see [14, Chapter 2]. In particular, for $k<s<k+1$, we have equivalent norms

$$
\|f\|_{B_{p}^{s}(\Omega)} \sim\|f\|_{H_{p}^{k}(\Omega)}+\left(\sum_{|\beta|=k} \int_{\Omega} \int_{\Omega} \frac{\left|D^{\beta} f(x)-D^{\beta} f(y)\right|^{p}}{|x-y|^{n+p(s-k)}} d x d y\right)^{1 / p} .
$$

For $s>0$, we define spaces $H_{p 0}^{s}(\Omega)$ and $B_{p 0}^{s}(\Omega)$ as the closures of $C_{\mathrm{c}}^{\infty}(\Omega)$ in $H_{p}^{s}(\Omega)$ and $B_{p}^{s}(\Omega)$, respectively. For negative $s \in \mathbb{R}$, we define $B_{p}^{s}(\Omega)$, $H_{p}^{s}(\Omega), B_{p 0}^{s}(\Omega)$ and $H_{p 0}^{s}(\Omega)$ as the dual spaces of $B_{p^{\prime} 0}^{-s}(\Omega), H_{p^{\prime} 0}^{-s}(\Omega)$, $B_{p^{\prime}}^{-s}(\Omega)$ and $H_{p^{\prime}}^{-s}(\Omega)$, respectively.

2.3. Function spaces on $\partial \Omega$. Let $\Omega$ be a bounded $C^{2}$-domain in $\mathbb{R}^{n}$, and put $\Delta(P, r)=B(P, r) \cap \partial \Omega$ for $P \in \partial \Omega$. Then, there is an $r_{0}>0$ such that, for each $P \in \partial \Omega$, there exists a bijective $C^{2}$ function $\Psi: B^{\prime}\left(0, r_{0}\right) \rightarrow \Delta\left(P, r_{0}\right)$, where $B^{\prime}\left(0, r_{0}\right)$ is the open ball in $\mathbb{R}^{n-1}$ whose radius is $r_{0}$ and whose center is the origin. Since $\Omega$ is bounded, there are $P_{1}, P_{2}, \ldots, P_{N}$ such that $\partial \Omega \subset \bigcup_{i=1}^{N} \Delta\left(P_{i}, r_{0}\right)$. Moreover, there exist bijective $C^{2}$-functions $\Psi_{i}: B^{\prime}\left(0, r_{0}\right) \rightarrow \Delta\left(P_{i}, r_{0}\right)$. Now, we say that $\phi$ is in the function space $H_{p}^{s}(\partial \Omega)$, for $-2 \leq s \leq 2$, if $\phi \circ \Psi_{i} \in H_{p}^{s}\left(B^{\prime}\left(0, r_{0}\right)\right)$ for all $1 \leq i \leq N$, and we equip this space with the norm

$$
\|\phi\|_{H_{p}^{s}(\partial \Omega)}:=\sum_{i=1}^{N}\left\|\phi \circ \Psi_{i}\right\|_{H_{p}^{s}\left(B^{\prime}\left(0, r_{0}\right)\right)} .
$$

Similarly, we define the function space $B_{p}^{s}(\partial \Omega)$. Clearly, for $0<s<2$, $H_{p}^{-s}(\partial \Omega)$ and $B_{p}^{-s}(\partial \Omega)$ are dual spaces of $H_{p^{\prime}}^{s}(\partial \Omega)$ and $B_{p^{\prime}}^{-s}(\partial \Omega)$, 
respectively. Again, for $0<\theta<1$,

$$
\begin{array}{r}
\left(H_{p}^{k_{0}}(\partial \Omega), H_{p}^{k_{1}}(\partial \Omega)\right)_{\theta, p}=B_{p}^{s}(\partial \Omega), \\
{\left[H_{p}^{k_{0}}(\partial \Omega), H_{p}^{k_{1}}(\partial \Omega)\right]_{\theta}=H_{p}^{s}(\partial \Omega),}
\end{array}
$$

where $s=(1-\theta) k_{0}+\theta k_{1}[\mathbf{1 4}$, Chapter 2].

We introduce the restriction theorem [15].

Proposition 2.1. Consider a bounded, Lipschitz domain $\Omega \subset B_{R}:=$ $B(0, R)$. For $0<s<\infty$ and $1<p<\infty$, the operator $\mathcal{R}$ : $B_{p}^{s+1 / p}\left(B_{R}\right) \rightarrow B_{p}^{s}(\partial \Omega)$ defined by $\mathcal{R}(F):=\left.F\right|_{\partial \Omega}$ is bounded, that is, there is a constant $c>0$, depending only on $n, s, \Omega$ and $R$, such that

$$
\|\mathcal{R}(F)\|_{B_{p}^{s}(\partial \Omega)} \leq c\|F\|_{B_{p}^{s+1 / p}\left(B_{R}\right)} .
$$

3. Boundary layer potential. The boundary integral operators associated with the fractional Laplacian and the classical Laplacian have the following properties.

Proposition 3.1. Let $\Omega$ be a bounded $C^{2}$ domain.

(1) For $-2 \leq s \leq 3-2 \alpha$ and $1<p<\infty$,

$$
S_{2 \alpha}: H_{p}^{s}(\partial \Omega) \longrightarrow H_{p}^{s+2 \alpha-1}(\partial \Omega)
$$

is a bounded operator.

(2) For $-1 \leq s \leq 0$,

$$
S_{2}: H_{p}^{s}(\partial \Omega) \longrightarrow H_{p}^{1+s}(\partial \Omega)
$$

is bijective.

Proof. See [19] for the proof of equation (3.1) (1) and [11] for equation (3.1) (2).

Let $\psi, \phi \in C^{2}(\partial \Omega)$, and consider the dual operator of (3.1), namely,

$$
S_{2 \alpha}^{*}: H_{p^{\prime}}^{-s-2 \alpha+1}(\partial \Omega) \longrightarrow H_{p^{\prime}}^{-s}(\partial \Omega) .
$$


Using (1.1), we have

$$
\begin{aligned}
\left\langle\left\langle S_{2 \alpha}^{*} \psi, \phi\right\rangle\right\rangle & =\left\langle\psi, S_{2 \alpha} \phi\right\rangle=\int_{\partial \Omega} \psi(P) S_{2 \alpha} \phi(P) d P \\
& =\int_{\partial \Omega} \phi(P) S_{2 \alpha} \psi(P) d P=\left\langle\phi, S_{2 \alpha} \psi\right\rangle,
\end{aligned}
$$

where $\langle\cdot, \cdot\rangle$ is the duality pairing between the spaces $H_{p}^{s+2 \alpha-1}(\partial \Omega)$ and $H_{p^{\prime}}^{-s-2 \alpha+1}(\partial \Omega)$, and where $\langle\langle\cdot, \cdot\rangle\rangle$ is the duality pairing between $H_{p}^{s}(\partial \Omega)$ and $H_{p^{\prime}}^{-s}(\partial \Omega)$. Since $C^{2}(\partial \Omega)$ is a dense subset of $H_{p}^{s}(\partial \Omega)$, equation (3.3) implies that, if $s<0$, then equation (3.2) is the same operator as

$$
S_{2 \alpha}: H_{p^{\prime}}^{-s-2 \alpha+1}(\partial \Omega) \longrightarrow H_{p^{\prime}}^{-s}(\partial \Omega)
$$

4. Proof of Theorem 1.1. To prove Theorem 1.1, we use the following proposition.

Proposition 4.1. Let $1 / 2<\alpha<1$. Given $\epsilon>0$, there are bounded linear operators $T^{1}: L^{p}(\partial \Omega) \rightarrow H_{p}^{1}(\partial \Omega)$ with $\left\|T^{1}\right\|_{L^{p}(\partial \Omega) \rightarrow H_{p}^{1}(\partial \Omega)}<\epsilon$ and $T^{2}: H_{p}^{-1}(\partial \Omega) \rightarrow H_{p}^{1}(\partial \Omega)$ such that

$$
S_{2 \alpha} S_{3-2 \alpha}=S_{2}+T^{1}+T^{2} .
$$

\section{Remark 4.2.}

(1) Since $S_{2}: L^{p}(\partial \Omega) \rightarrow H_{p}^{1}(\partial \Omega)$ is bijective, for sufficiently small $\epsilon>$ 0 , it follows that $S_{2}+T^{1}: L^{p}(\partial \Omega) \rightarrow H_{p}^{1}(\partial \Omega)$ is also bijective.

(2) Since each of $S_{2}, S_{2 \alpha} S_{3-2 \alpha}$ and $T^{2}$ is bounded from $H_{p}^{-1}(\partial \Omega)$ to $L^{p}(\partial \Omega)$, the operator $T^{1}: H_{p}^{-1}(\partial \Omega) \rightarrow L^{p}(\partial \Omega)$ is also bounded. Then, from the complex interpolation property (2.1), we obtain that, for $-1<s<0$,

$$
\left\|T^{1}\right\|_{H_{p}^{s}(\partial \Omega) \rightarrow H_{p}^{1+s}(\partial \Omega)} \leq c \epsilon^{1+s}
$$

(3) From the arguments of (1) and (2) above, and by Proposition 3.1, we conclude that $S_{2}+T^{1}: H_{p}^{s}(\partial \Omega) \rightarrow H_{p}^{1+s}(\partial \Omega)$ is bijective for $-1<s<0$.

Proof of Proposition 4.1. Let $0<15 \epsilon<r_{0}$, where $r_{0}>0$ is defined in subsection 2.3. Let $P_{1}, P_{2}, \ldots, P_{m} \in \partial \Omega$ be such that $\left|P_{i}-P_{j}\right|>\epsilon$ 
and $\partial \Omega \subset \bigcup_{i=1}^{m} B\left(P_{i}, \epsilon\right)$. Let $\left\{\eta_{i}\right\},\left\{\kappa_{i}\right\}$ and $\left\{\lambda_{i}\right\}$ be partitions of the unity of $\left\{B\left(P_{i}, 2 \epsilon\right)\right\},\left\{B\left(P_{i}, 7 \epsilon\right)\right\}$ and $\left\{B\left(P_{i}, 12 \epsilon\right)\right\}$, respectively, such that

$$
\begin{aligned}
& \operatorname{supp} \eta_{i} \subset B\left(P_{i}, 2 \epsilon\right), \quad \eta_{i} \equiv 1 \text { in } B\left(P_{i}, \epsilon\right) \text {, } \\
& \operatorname{supp} \kappa_{i} \subset B\left(P_{i}, 7 \epsilon\right), \quad \kappa_{i} \equiv 1 \text { in } B\left(P_{i}, 5 \epsilon\right) \text {, } \\
& \operatorname{supp} \lambda_{i} \subset B\left(P_{i}, 12 \epsilon\right), \quad \eta_{i} \equiv 1 \text { in } B\left(P_{i}, 10 \epsilon\right) \text {. }
\end{aligned}
$$

Then, for $\phi \in L^{p}(\partial \Omega)$, we have $S_{2 \alpha} S_{3-2 \alpha} \phi=I_{1} \phi+I_{2} \phi$, where

$$
I_{1} \phi:=\sum_{i} \eta_{i} S_{2 \alpha} \kappa_{i} S_{3-2 \alpha} \phi
$$

and

$$
I_{2} \phi:=\sum_{i} \eta_{i} S_{2 \alpha}\left(1-\kappa_{i}\right) S_{3-2 \alpha} \phi
$$

Note that, since $\operatorname{supp} \eta_{i} \subset B\left(P_{i}, 2 \epsilon\right)$ and $\kappa_{i} \equiv 1$ in $\Delta\left(P_{i}, 5 \epsilon\right)$, it follows that the kernel of the boundary integral operator $\eta_{i} S_{2 \alpha}\left(1-\kappa_{i}\right)$ has no singularity in $\partial \Omega$, and so $\eta_{i} S_{2 \alpha}\left(1-\kappa_{i}\right): H_{p}^{-1}(\partial \Omega) \rightarrow H_{p}^{1}(\partial \Omega)$ is bounded. Since $S_{3-2 \alpha}: H_{p}^{-1}(\partial \Omega) \rightarrow H_{p}^{1-2 \alpha}(\partial \Omega)$ is bounded (see Proposition 3.1), so is $I_{2}: H_{p}^{-1}(\partial \Omega) \rightarrow H_{p}^{1}(\partial \Omega)$. have

For $I_{1} \phi$, since supp $\lambda_{i} \subset B\left(P_{i}, 12 \epsilon\right)$ and $\lambda_{i} \equiv 1$ in $B\left(P_{i}, 10 \epsilon\right)$, we

$$
I_{1} \phi=I_{11} \phi+I_{12} \phi, \quad I_{11} \phi:=\sum_{i} I_{11}^{i} \phi, \quad I_{12} \phi:=\sum_{i} I_{12}^{i},
$$

where

$$
\begin{aligned}
I_{11} \phi^{i}(P):=\eta_{i} S_{2 \alpha} \kappa_{i} S_{3-2 \alpha} \lambda_{i} \phi(P) & \\
= & \eta_{i}(P) \int_{\partial \Omega} \Gamma_{2 \alpha}(P-Z) \kappa_{i}(Z) \\
& \int_{\Delta\left(P_{i}, 12 \epsilon\right)} \lambda_{i}(Q) \Gamma_{3-2 \alpha}(Z-Q) \phi(Q) d Q d Z
\end{aligned}
$$


and

$$
\begin{gathered}
I_{12} \phi(P):=\eta_{i} S_{2 \alpha} \kappa_{i} S_{3-2 \alpha}\left(1-\lambda_{i}\right) \phi(P) \\
=\eta_{i}(P) \int_{\partial \Omega} \Gamma_{2 \alpha}(P-Z) \kappa_{i}(Z) \\
\int_{\partial \Omega \backslash \Delta\left(P_{i}, 10 \epsilon\right)}\left(1-\lambda_{i}(Q)\right) \Gamma_{3-2 \alpha}(Z-Q) \phi(Q) d Q d Z .
\end{gathered}
$$

Since $\operatorname{supp} \kappa_{i} \subset B\left(P_{i}, 7 \epsilon\right)$ and $\lambda_{i} \equiv 1$ in $B\left(P_{i}, 10 \epsilon\right)$, it follows that the kernel of $\kappa_{i} S_{3-2 \alpha}\left(1-\lambda_{i}\right)$ has no singularity in $\partial \Omega$ and so the operator $\kappa_{i} S_{3-2 \alpha}\left(1-\lambda_{i}\right): H_{p}^{-1}(\partial \Omega) \rightarrow H_{p}^{1}(\partial \Omega)$ is bounded. Hence, from Proposition 3.1, $I_{12}: H_{p}^{-1}(\partial \Omega) \rightarrow H_{p}^{1}(\partial \Omega)$ is a bounded operator.

Similarly, we decompose $S_{2} \phi$ into

$$
S_{2} \phi(P)=J_{11} \phi(P)+J_{12} \phi(P)+J_{2} \phi(P),
$$

where $J_{12}, J_{2}: H_{p}^{-1}(\partial \Omega) \rightarrow H_{p}^{1}(\partial \Omega)$ are bounded operators and where $J_{11} \phi:=\sum_{i} J_{11}^{i} \phi$ for

$$
J_{11}^{i} \phi(P):=\eta_{i}(P) \int_{\Delta\left(P_{i}, 12 \epsilon\right)} \Gamma_{2}(P-Q) \lambda_{i}(Q) \phi(Q) d Q .
$$

For $I_{11} \phi$ and $J_{11} \phi$, we fix $i$. After translation and rotation, we may assume that $P_{i}=0$, and there is $\Psi_{i}: B^{\prime}(0,15 \epsilon) \rightarrow \mathbb{R}$ with

$$
\begin{aligned}
\left|\Psi_{i}\left(x^{\prime}\right)\right|<c\left|x^{\prime}\right|^{2}<c \epsilon^{2} & \text { and } \\
\left|\nabla \Psi_{i}\left(x^{\prime}\right)\right|<c\left|x^{\prime}\right|<c \epsilon & \text { for } x^{\prime} \in B^{\prime}(0,15 \epsilon),
\end{aligned}
$$

such that, for $Q \in \Delta_{15 \epsilon}^{i}:=\Delta\left(P_{i}, 15 \epsilon\right)$, the point $Q$ is represented by $Q=\left(y^{\prime}, \Psi\left(y^{\prime}\right)\right)$ for some $y^{\prime} \in B^{\prime}(15 \epsilon):=B^{\prime}(0,15 \epsilon)$. Let $P=\left(x^{\prime}, \Psi\left(x^{\prime}\right)\right)$ for $x^{\prime} \in B^{\prime}(0,2 \epsilon)$. Then, we have

$$
\begin{aligned}
I_{11}^{i} \phi(P)= & \eta_{i}(P) \int_{\Delta_{12 \epsilon}^{i}} \lambda_{i}(Q) \phi(Q) \\
& \int_{\partial \Omega} \kappa_{i}(Z) \Gamma_{2 \alpha}(P-Z) \Gamma_{3-2 \alpha}(Q-Z) d Z d Q \\
= & \eta_{i}(P) \int_{B^{\prime}(12 \epsilon)} \lambda_{i}\left(y^{\prime}, \Psi\left(y^{\prime}\right)\right) \phi\left(y^{\prime}, \Psi\left(y^{\prime}\right)\right) \sqrt{1+\left|\nabla \Psi\left(y^{\prime}\right)\right|^{2}} \\
& \int_{B^{\prime}(7 \epsilon)} \kappa_{i}\left(z^{\prime}, \Psi\left(z^{\prime}\right)\right) \Gamma_{2 \alpha}\left(x^{\prime}-z^{\prime}, \Psi\left(x^{\prime}\right)-\Psi\left(z^{\prime}\right)\right)
\end{aligned}
$$




$$
\Gamma_{3-2 \alpha}\left(y^{\prime}-z^{\prime}, \Psi\left(y^{\prime}\right)-\Psi\left(z^{\prime}\right)\right) \sqrt{1+\left|\nabla \Psi\left(z^{\prime}\right)\right|^{2}} d z^{\prime} d y^{\prime}
$$

and

$$
\begin{aligned}
& J_{11}^{i} \phi(P)=\eta_{i}(P) \int_{B^{\prime}(12 \epsilon)} \Gamma_{2}\left(x^{\prime}-y^{\prime}, \Psi\left(x^{\prime}\right)-\Psi\left(y^{\prime}\right)\right) \\
& \lambda_{i}\left(y^{\prime}, \Psi\left(y^{\prime}\right)\right) \phi\left(y^{\prime}, \Psi\left(y^{\prime}\right)\right) \sqrt{1+\left|\nabla \Psi\left(y^{\prime}\right)\right|^{2}} d y^{\prime} .
\end{aligned}
$$

In the Appendix, we prove that the quantities

$$
\begin{aligned}
I_{111}^{i} \phi(P):= & \eta_{i}(P) \int_{B^{\prime}(12 \epsilon)} \lambda_{i}\left(y^{\prime}, 0\right) \phi\left(y^{\prime}, \Psi\left(y^{\prime}\right)\right) \\
& \int_{B^{\prime}(7 \epsilon)} \kappa_{i}\left(z^{\prime}, 0\right) \Gamma_{2 \alpha}\left(x^{\prime}-z^{\prime}, 0\right) \Gamma_{3-2 \alpha}\left(y^{\prime}-z^{\prime}, 0\right) d z^{\prime} d y^{\prime}
\end{aligned}
$$

and

$$
J_{111}^{i} \phi(P):=\eta_{i}(P) \int_{B^{\prime}(12 \epsilon)} \lambda_{i}\left(y^{\prime}, 0\right) \Gamma_{2}\left(x^{\prime}-y^{\prime}, 0\right) \phi\left(y^{\prime}, \Psi\left(y^{\prime}\right)\right) d y^{\prime}
$$

satisfy the inequalities

$$
\begin{aligned}
\left\|I_{11}^{i}-I_{111}^{i}\right\|_{L^{p}\left(\Delta_{12 \epsilon}^{i}\right) \rightarrow H_{p}^{1}\left(\Delta_{2 \epsilon}^{i}\right)} & \leq c \epsilon, \\
\left\|J_{11}^{i}-J_{111}^{i}\right\|_{L^{p}\left(\Delta_{12 \epsilon}^{i}\right) \rightarrow H_{p}^{1}\left(\Delta_{2 \epsilon}^{i}\right)} & \leq c \epsilon .
\end{aligned}
$$

It is well known [19, Section 5.1] that

$$
\int_{\mathbb{R}^{n-1}} \Gamma_{2 \alpha}\left(x^{\prime}-z^{\prime}, 0\right) \Gamma_{3-2 \alpha}\left(y^{\prime}-z^{\prime}, 0\right) d z^{\prime}=\Gamma_{2}\left(x^{\prime}-y^{\prime}, 0\right) .
$$

Hence, we have

$$
\begin{aligned}
\Gamma_{2}\left(x^{\prime}-\right. & \left.y^{\prime}, 0\right)=\int_{\mathbb{R}^{n-1}} \kappa_{i}\left(z^{\prime}, 0\right) \Gamma_{2 \alpha}\left(x^{\prime}-z^{\prime}, 0\right) \Gamma_{3-2 \alpha}\left(y^{\prime}-z^{\prime}, 0\right) d z^{\prime} \\
& +\int_{\mathbb{R}^{n-1}}\left(1-\kappa_{i}\left(z^{\prime}, 0\right)\right) \Gamma_{2 \alpha}\left(x^{\prime}-z^{\prime}, 0\right) \Gamma_{3-2 \alpha}\left(y^{\prime}-z^{\prime}, 0\right) d z^{\prime} \\
= & \int_{\mathbb{R}^{n-1}} \kappa_{i}\left(z^{\prime}, 0\right) \Gamma_{2 \alpha}\left(x^{\prime}-z^{\prime}, 0\right) \Gamma_{3-2 \alpha}\left(y^{\prime}-z^{\prime}, 0\right) d z^{\prime}+k_{i}\left(x^{\prime}, y^{\prime}\right),
\end{aligned}
$$

where

$$
k_{i}\left(x^{\prime}, y^{\prime}\right):=\int_{\mathbb{R}^{n-1}}\left(1-\kappa_{i}\left(z^{\prime}, 0\right)\right) \Gamma_{2 \alpha}\left(x^{\prime}-z^{\prime}, 0\right) \Gamma_{3-2 \alpha}\left(y^{\prime}-z^{\prime}, 0\right) d z^{\prime} .
$$


Note that, for $x^{\prime} \in B^{\prime}(2 \epsilon)$ the kernel $k_{i}\left(x^{\prime}, y^{\prime}\right)$ has no singularity with respect to $x^{\prime}$. Thus,

$$
I_{111}^{i} \phi(P)-J_{111}^{i} \phi(P)=\eta_{i}(P) \int_{B^{\prime}(12 \epsilon)} \lambda_{i}\left(y^{\prime}, 0\right) \phi\left(y^{\prime}, \Psi\left(y^{\prime}\right)\right) k_{i}\left(x^{\prime}, y^{\prime}\right) d y^{\prime}
$$

is a smooth function of $P \in \partial \Omega$. Let

$$
T^{1}:=\sum_{i}\left(I_{11}^{i}-I_{111}^{i}\right)+\sum_{i}\left(J_{11}^{i}-J_{111}^{i}\right)
$$

and

$$
T_{2}:=I_{2}+J_{2}+I_{12}+J_{12}+\sum_{i}\left(I_{111}^{i}-J_{111}^{i}\right) .
$$

Then, $S_{2 \alpha} S_{3-2 \alpha}=S_{2}+T^{1}+T^{2}$ is such that $T^{2}$ has a smooth kernel, and

$$
\begin{aligned}
\left\|T^{1} \phi\right\|_{H_{p}^{1}(\partial \Omega)} & \leq c \sum_{i}\left(\left\|\left(I_{11}^{i}-I_{111}^{i}\right) \phi\right\|_{H_{p}^{1}(\partial \Omega)}+\left\|\left(J_{11}^{i}-J_{111}^{i}\right) \phi\right\|_{H_{p}^{1}(\partial \Omega)}\right) \\
& \leq c \epsilon \sum_{i}\|\phi\|_{L^{p}\left(\Delta_{12 \epsilon}^{i}\right)} \leq c \epsilon\|\phi\|_{L^{p}(\partial \Omega)},
\end{aligned}
$$

completing the proof of Proposition 4.1.

Let $p_{0}:=2(n-1) /(n-2+2 \alpha)$, and note that $p_{0}<2$.

Proof of Theorem 1.1. $p \geq p_{0}$. To show the injectivity, suppose that $S_{2 \alpha} \phi=0$ for $\phi \in L^{p}(\partial \Omega)$. From the Hölder inequality and Sobolev imbedding, $L^{p}(\partial \Omega) \subset L^{p_{0}}(\partial \Omega) \subset H_{2}^{-\alpha+1 / 2}(\partial \Omega)$. Since $S_{2 \alpha}$ : $H_{2}^{-\alpha+1 / 2}(\partial \Omega) \rightarrow H_{2}^{\alpha-1 / 2}(\partial \Omega)$ is bijective [6], we have $\phi=0$. Thus, $S_{2 \alpha}: L^{p}(\partial \Omega) \rightarrow H_{p}^{2 \alpha-1}(\partial \Omega)$ is injective for $p \geq p_{0}$.

To show that $S_{2 \alpha}: L^{p}(\partial \Omega) \rightarrow H_{p}^{2 \alpha-1}(\partial \Omega)$ is surjective, let $f \in$ $H_{p}^{2 \alpha-1}(\partial \Omega)$. Based on Sobolev imbedding and the Hölder inequality, $H_{p}^{2 \alpha-1}(\partial \Omega) \subset H_{p_{0}}^{2 \alpha-1}(\partial \Omega) \subset H_{2}^{\alpha-1 / 2}(\partial \Omega)$. From the bijectivity of $S_{2 \alpha}$ : $H_{2}^{-\alpha+1 / 2}(\partial \Omega) \rightarrow H_{2}^{\alpha-1 / 2}(\partial \Omega)$, there exist $\phi \in H_{2}^{-\alpha+1 / 2}(\partial \Omega)$ such that $S_{2 \alpha} \phi=f$.

Note that, from Proposition 4.1, we determine that $S_{3-2 \alpha} S_{2 \alpha}=S_{2}+$ $T^{1}+T^{2}$, where $\left\|T^{1}\right\|_{L^{p}(\partial \Omega) \rightarrow H_{p}^{1}(\partial \Omega)} \leq \epsilon$ and $T^{2}: H_{p}^{-1}(\partial \Omega) \rightarrow H_{p}^{1}(\partial \Omega)$ is 
bounded. Since $f \in H_{p}^{2 \alpha-1}(\partial \Omega)$, it follows that $S_{3-2 \alpha} S_{2 \alpha} \phi=S_{3-2 \alpha} f \in$ $H_{p}^{1}(\partial \Omega)$.

Then, from Proposition 4.1, we obtain that $\left(S_{2}+T^{1}\right) \phi=S_{3-2 \alpha} S_{2 \alpha} \phi-$ $T^{2} \phi \in H_{p}^{1}(\partial \Omega)$. Considering $\epsilon>0$ sufficiently small that $S_{2}+T^{1}$ : $L^{p}(\partial \Omega) \rightarrow H_{p}^{1}(\partial \Omega)$ is bijective, see Remark $4.2(1)$, we obtain that $\phi \in L^{p}(\partial \Omega)$. This implies that $S_{2 \alpha}: L^{p}(\partial \Omega) \rightarrow H_{p}^{2 \alpha-1}(\partial \Omega)$ is surjective. Hence, the proof of the bijectivity of $S_{2 \alpha}: L^{p}(\partial \Omega) \rightarrow H_{p}^{2 \alpha-1}(\partial \Omega)$ for $p \geq p_{0}$ is complete.

\section{Remark 4.3.}

(1) The dual operator

$$
S_{2 \alpha}^{*}: H_{p^{\prime}}^{-2 \alpha+1}(\partial \Omega) \longrightarrow L^{p^{\prime}}(\partial \Omega)
$$

of $S_{2 \alpha}: L^{p}(\partial \Omega) \rightarrow H_{p}^{2 \alpha-1}(\partial \Omega)$ is the same as the operator $S_{2 \alpha}$ : $H_{p^{\prime}}^{-2 \alpha+1}(\partial \Omega) \rightarrow L^{p^{\prime}}(\partial \Omega)$, where $1 / p+1 / p^{\prime}=1$, by Section 3 . Hence, from the property of the dual operator, $S_{2 \alpha}: H_{p}^{-2 \alpha+1}(\partial \Omega) \rightarrow L^{p}(\partial \Omega)$ is bijective for $1<p \leq p_{0}^{\prime}=2(n-1) /(n-2 \alpha)$.

(2) In Proposition 4.1, $S_{3-2 \alpha} S_{2 \alpha}$ is the sum of a bijective operator $S_{2}+T^{1}$ and a compact operator $T^{2}$, so $S_{3-2 \alpha} S_{2 \alpha}$ is a Fredholm operator with index zero. Since $S_{2 \alpha}: L^{p}(\partial \Omega) \rightarrow H_{p}^{2 \alpha-1}(\partial \Omega)$ and $S_{3-2 \alpha}$ : $H_{p}^{2 \alpha-1}(\partial \Omega) \rightarrow H_{p}^{1}(\partial \Omega)$ are injective, it follows that $S_{3-2 \alpha} S_{2 \alpha}$ is injective, and so by the Fredholm operator theorem, $S_{3-2 \alpha} S_{2 \alpha}: L^{p}(\partial \Omega) \rightarrow$ $H_{p}^{1}(\partial \Omega)$ is bijective. This implies that $S_{3-2 \alpha}: H_{p}^{2 \alpha-1}(\partial \Omega) \rightarrow H_{p}^{1}(\partial \Omega)$ is bijective for $p \geq p_{0}=2(n-1) /(n-2+2 \alpha)$.

Proof of Theorem 1.1. $1<p<2$. Now, we will show that $S_{2 \alpha}$ : $H_{p^{\prime}}^{-2 \alpha+1}(\partial \Omega) \rightarrow L^{p^{\prime}}(\partial \Omega)$ is surjective. Let $f \in L^{p^{\prime}}(\partial \Omega)$. Based on the Hölder inequality, $L^{p^{\prime}}(\partial \Omega) \subset L^{2}(\partial \Omega)$ and, from the bijectivity of $S_{2 \alpha}: H_{2}^{-2 \alpha+1}(\partial \Omega) \rightarrow L^{2}(\partial \Omega)$, see Remark 4.3 (1), there exists $\phi \in H_{2}^{-2 \alpha+1}(\partial \Omega)$ such that $S_{2 \alpha} \phi=f$. Then, $S_{3-2 \alpha} S_{2 \alpha} \phi=S_{3-2 \alpha} f \in$ $H_{q}^{2-2 \alpha}(\partial \Omega)$. Since $T^{2} \phi \in H_{1}^{1}(\partial \Omega) \subset H_{p^{\prime}}^{2-2 \alpha}(\partial \Omega)$ based on Proposition 4.1 ,

$$
\left(S_{2}+T^{1}\right) \phi=S_{3-2 \alpha} S_{2 \alpha} \phi-T^{2} \phi \in H_{p^{\prime}}^{2-2 \alpha}(\partial \Omega) .
$$


Since $S_{2}+T^{1}: H_{p^{\prime}}^{-2 \alpha+1}(\partial \Omega) \rightarrow H_{p^{\prime}}^{2-2 \alpha}(\partial \Omega)$ is bijective, see Remark 4.2 (3), we obtain that $\phi \in H_{p^{\prime}}^{-2 \alpha+1}(\partial \Omega)$. This implies that $S_{2 \alpha}$ : $H_{p^{\prime}}^{-2 \alpha+1}(\partial \Omega) \rightarrow L^{p^{\prime}}(\partial \Omega)$ is surjective.

Based on the dual operator property, $S_{2 \alpha}^{*}: L^{p}(\partial \Omega) \rightarrow H_{p}^{2 \alpha-1}(\partial \Omega)$ is injective for $1<p<2$. Note that $S_{2 \alpha}^{*}=S_{2 \alpha}$. Since $S_{2 \alpha}: L^{p}(\partial \Omega) \rightarrow$ $H_{p}^{2 \alpha-1}(\partial \Omega)$ is injective, so is $S_{2 \alpha}: H_{p}^{2 \alpha-1}(\partial \Omega) \rightarrow H_{p}^{1}(\partial \Omega)$. Hence, $S_{2 \alpha} S_{3-2 \alpha}: L^{p}(\partial \Omega) \rightarrow H_{p}^{1}(\partial \Omega)$ is injective for $1<p<2$.

In Remark 4.2 (3), $S_{2 \alpha} S_{3-2 \alpha}$ is the sum of a bijective operator and a compact operator. Hence, by the Fredholm theorem, $S_{2 \alpha} S_{3-2 \alpha}$ : $L^{p}(\partial \Omega) \rightarrow H_{p}^{1}(\partial \Omega)$ is bijective.

To show $S_{2 \alpha}: L^{p}(\partial \Omega) \rightarrow H_{p}^{2 \alpha-1}(\partial \Omega)$ is surjective for $1<p<2$, let $f \in H_{p}^{2 \alpha-1}(\partial \Omega)$. Therefore, $S_{3-2 \alpha} f \in H_{p}^{1}(\partial \Omega)$. Since $S_{3-2 \alpha} S_{2 \alpha}$ : $L^{p}(\partial \Omega) \rightarrow H_{p}^{1}(\partial \Omega)$ is bijective, there is a $\phi \in L^{p}(\partial \Omega)$ such that $S_{3-2 \alpha} S_{2 \alpha} \phi=S_{3-2 \alpha} f$. Since $S_{3-2 \alpha}$ is injective, $S_{2 \alpha} \phi=f$, and so $S_{2 \alpha}: L^{p}(\partial \Omega) \rightarrow H_{p}^{2 \alpha-1}(\partial \Omega)$ is bijective.

Corollary 4.4. Let $1 / 2<\alpha<1$ and $1<p<\infty$. Then the following operators are bijective:

$$
\begin{array}{ll}
S_{2 \alpha}: H_{p}^{s}(\partial \Omega) \longrightarrow H_{p}^{s+2 \alpha-1}(\partial \Omega) & \text { for }-1 \leq s \leq 2-2 \alpha, \\
S_{2 \alpha}: B_{p}^{s}(\partial \Omega) \longrightarrow B_{p}^{s+2 \alpha-1}(\partial \Omega) & \text { for }-1<s<2-2 \alpha .
\end{array}
$$

Proof. In the proof of Theorem 1.1, $S_{3-2 \alpha}: L^{p}(\partial \Omega) \rightarrow H_{p}^{2-2 \alpha}(\partial \Omega)$ and $S_{2 \alpha}: H_{p}^{2-2 \alpha}(\partial \Omega) \rightarrow H_{p}^{1}(\partial \Omega)$ are injective, and so $S_{2 \alpha} S_{3-2 \alpha}:$ $L^{p}(\partial \Omega) \rightarrow H_{p}^{1}(\partial \Omega)$ is injective. Since $S_{2 \alpha} S_{3-2 \alpha}: L^{p}(\partial \Omega) \rightarrow H_{p}^{1}(\partial \Omega)$ is the Fredholm operator with index $0, S_{2 \alpha} S_{3-2 \alpha}: L^{p}(\partial \Omega) \rightarrow H_{p}^{1}(\partial \Omega)$ is bijective. This implies that

$$
S_{2 \alpha}: H_{p}^{2-2 \alpha}(\partial \Omega) \rightarrow H_{p}^{1}(\partial \Omega) \quad \text { is bijective. }
$$

From the dual operator property and the fact that $S_{2 \alpha}^{*}=S_{2 \alpha}$,

$$
S_{2 \alpha}: H_{p}^{-1}(\partial \Omega) \longrightarrow H_{p}^{-2+2 \alpha}(\partial \Omega) \text { is bijective. }
$$

Using (4.5), (4.6) and the properties of real and complex interpolation, we obtain the corollary. 
5. Proof of Theorem 1.2. We introduce the Riesz potential $I_{2 \alpha}$, defined for $0<2 \alpha<n$, by

$$
I_{2 \alpha} f(x):=c(n, \alpha) \int_{\mathbb{R}^{n}} \frac{f(y) d y}{|x-y|^{n-2 \alpha}} \quad \text { for } \psi \in C_{\mathrm{c}}^{\infty}\left(\mathbb{R}^{n}\right),
$$

where

$$
c(n, \alpha):=\frac{(2 \pi)^{2 \alpha} \Gamma((1 / 2) n-\alpha)}{\pi^{n / 2} 2^{2 \alpha} \Gamma(\alpha)} .
$$

The results in the next two propositions are well known [19, Chapter 5] and will be useful in subsequent estimates.

Proposition 5.1. The Riesz potential is a bounded linear operator

$$
I_{2 \alpha}: L^{p}\left(\mathbb{R}^{n}\right) \rightarrow L^{q}\left(\mathbb{R}^{n}\right) \quad \text { for } 1<p<q<\infty \text { and } \frac{1}{q}=\frac{1}{p}-\frac{2 \alpha}{n} .
$$

Proposition 5.2. For $1<p<\infty$ and $s \in \mathbb{R}$, the Riesz potential defines bounded linear operators

$$
I_{2 \alpha}: H_{p}^{s}\left(\mathbb{R}^{n}\right) \longrightarrow H_{p}^{s+2 \alpha}\left(\mathbb{R}^{n}\right)
$$

and

$$
I_{2 \alpha}: B_{p}^{s}\left(\mathbb{R}^{n}\right) \longrightarrow B_{p}^{s+2 \alpha}\left(\mathbb{R}^{n}\right)
$$

Remark 5.3. Let $B_{R}$ be the open ball in $\mathbb{R}^{n}$ with radius $R$, centered at the origin, and put

$$
\widetilde{I}_{2 \alpha} f(x)=\int_{\mathbb{R}^{n}} \Gamma_{2 \alpha}(x-y) f(y) d y .
$$

Then, based on Proposition 5.2,

$$
\widetilde{I}_{2 \alpha}: B_{p 0}^{s}\left(B_{R}\right) \rightarrow B_{p}^{s+2 \alpha}\left(B_{R}\right) \text { is bounded for } s \in \mathbb{R} .
$$

Proof of equation (1.4). Let $-2 \alpha+1-1 / p<s<0, \phi \in C^{1}(\partial \Omega)$ and $f \in C_{\mathrm{c}}^{\infty}\left(B_{R}\right)$. Then, we have

$$
\int_{\mathbb{R}^{n}} f(x) \mathcal{S}_{2 \alpha} \phi(x) d x=\int_{\partial \Omega} \phi(P) \widetilde{I}_{2 \alpha} f(P) d P .
$$


Since $C^{1}(\partial \Omega)$ is a dense subspace of $B_{p}^{s}(\partial \Omega)$, and since $C_{\mathrm{c}}^{\infty}\left(B_{R}\right)$ is a dense subspace of $B_{p^{\prime} 0}^{-s-2 \alpha+1 / p^{\prime}}\left(B_{R}\right)$,

$$
\left\langle f, \mathcal{S}_{2 \alpha} \phi\right\rangle_{\left(B_{p^{\prime} 0}^{-s-2 \alpha+1 / p^{\prime}}\left(B_{R}\right), B_{p}^{s+2 \alpha-1+1 / p}\left(B_{R}\right)\right)}=\left\langle\phi, \widetilde{I}_{2 \alpha} f\right\rangle_{\left(B_{p}^{s}(\partial \Omega), B_{p^{\prime}}^{-s}(\partial \Omega)\right)} .
$$

Here, $\langle\cdot, \cdot\rangle_{\left(X^{\prime}, X\right)}$ denotes the duality pairing between a Banach space $X$ and its dual space $X^{\prime}$. Then, by Propositions 2.1 and 5.2, we have

$$
\begin{gathered}
\left\langle f, \mathcal{S}_{2 \alpha} \phi\right\rangle_{\left(B_{p^{\prime} 0}^{-s-2 \alpha+1 / p^{\prime}}\left(B_{R}\right), B_{p}^{s+2 \alpha-1+1 / p}\left(B_{R}\right)\right)} \leq\|\phi\|_{B_{p}^{s}(\partial \Omega)} \\
\left\|\widetilde{I}_{2 \alpha} f\right\|_{B_{p^{\prime}}^{-s}(\partial \Omega)} \leq c\|\phi\|_{B_{p}^{s}(\partial \Omega)}\left\|\widetilde{I}_{2 \alpha} f\right\|_{B_{p^{\prime}}^{-s+1 / p^{\prime}}\left(B_{R}\right)} \\
\leq c\|\phi\|_{B_{p}^{s}(\partial \Omega)}\|f\|_{B_{p^{\prime} 0}^{-s-2 \alpha+1 / p^{\prime}}\left(B_{R}\right)} .
\end{gathered}
$$

Hence,

$$
\left\|\mathcal{S}_{2 \alpha} \phi\right\|_{B_{p}^{s+2 \alpha-1+1 / p}\left(B_{R}\right)} \leq c\|\phi\|_{B_{p}^{s}(\partial \Omega)}
$$

which completes the proof of equation (1.4).

Proof of equation (1.5). For $\phi \in B_{p}^{s}(\partial \Omega)$ and $-2 \alpha+1-1 / p<s<0$, let $u$ be the layer potential of $\phi$ defined by equation (1.3). Note that $u$ is in $C^{\infty}\left(\mathbb{R}^{n} \backslash \partial \Omega\right)$ and, for large $|x|$, we have

$$
\left|D^{\beta} u(x)\right| \leq\|\phi\|_{B_{p}^{s}(\partial \Omega)}\left\|D^{\beta} \Gamma_{2 \alpha}(x-\cdot)\right\|_{B_{p^{\prime}}^{-s}(\partial \Omega)} \leq \frac{c\|\phi\|_{B_{p}^{s}(\partial \Omega)}}{|x|^{n-2 \alpha+|\beta|}} .
$$

Let $B_{R}$ be an open ball whose center is the origin and the radius is $R \geq 2$, such that $\Omega \subset B_{R / 3}$. We divide $|u|_{B_{p}^{s+2 \alpha-1+1 / p}}^{p}$ into three parts:

$$
\begin{aligned}
& A_{1}:=\int_{|x| \leq R} \int_{|y| \leq R} \frac{\left|D^{k} u(x)-D^{k} u(y)\right|^{p}}{|x-y|^{n+p(s+2 \alpha-k-1+1 / p)}} d y d x \\
& A_{2}:=2 \int_{|x| \leq R} \int_{|y| \geq R} \frac{\left|D^{k} u(x)-D^{k} u(y)\right|^{p}}{|x-y|^{n+p(s+2 \alpha-k-1+1 / p)}} d y d x \\
& A_{3}:=\int_{|x| \geq R} \int_{|y| \geq R} \frac{\left|D^{k} u(x)-D^{k} u(y)\right|^{p}}{|x-y|^{n+p(s+2 \alpha-k-1+1 / p)}} d y d x .
\end{aligned}
$$

From equation (1.4), $A_{1}$ is dominated by $\|\phi\|_{B_{p}^{s}(\partial \Omega)}^{p}$. For $|x| \leq R$ and $|y| \geq 2 R$, we determine that $|x-y| \geq|y|-|x| \geq|y|-R \geq|y| / 2$. Note that, from equation (5.1), $\left|D^{k} u(y)\right| \leq c|y|^{-n+2 \alpha-k}\|\phi\|_{B_{p}^{s}(\partial \Omega)}^{2}$ for 
$|y| \geq 2 R$. Hence, from equation (1.4),

$$
\begin{aligned}
A_{2} \leq & 2 \int_{|x| \leq R} \int_{R \leq|y| \leq 2 R} \frac{\left|D^{k} u(x)-D^{k} u(y)\right|^{p}}{|x-y|^{n+p(s+2 \alpha-k-1+1 / p)}} d y d x \\
& +2^{n+2 s+2} \int_{|x| \leq R} \int_{|y| \geq 2 R} \frac{\left|D^{k} u(x)\right|^{p}+\left|D^{k} u(y)\right|^{p}}{|y|^{n+p(s+2 \alpha-k-1+1 / p)}} d y d x \\
\leq & c_{R}\|u\|_{B_{p}^{s+2 \alpha-1+1 / p}(B(2 R))}^{p} \\
& +c\|\phi\|_{B_{p}^{s}(\partial \Omega)}^{p} \int_{|x| \leq R} \int_{|y| \geq 2 R} \frac{d y d x}{|y|^{n+p(s-1+n+1 / p)}},
\end{aligned}
$$

and thus, $A_{2} \leq c_{R}\|\phi\|_{B_{p}^{s}(\partial \Omega)}^{p}$. We divide $A_{3}$ into two parts:

$$
\begin{aligned}
A_{3}= & \int_{|x| \geq R} \int_{|y| \geq R,|x-y| \leq|x| / 2} \frac{\left|D^{k} u(x)-D^{k} u(y)\right|^{p}}{|x-y|^{n+p(s+2 \alpha-k-1+1 / p)}} d y d x \\
& +\int_{|x| \geq R} \int_{|y| \geq R,|x-y| \geq|x| / 2} \frac{\left|D^{k} u(x)-D^{k} u(y)\right|^{p}}{|x-y|^{n+p(s+2 \alpha-k-1+1 / p)}} d y d x
\end{aligned}
$$

Applying the mean-value theorem, for $|x| \geq R,|x-y| \leq|x| / 2$, there is a $\xi$ between $x$ and $y$ such that $D^{k} u(x)-D^{k} u(y)=D^{k+1} u(\xi) \cdot(x-y)$. Note that $|x-\xi| \leq|x| / 2$, and hence $|\xi| \geq|x| / 2 \geq R / 2$. Since $s+2 \alpha-k-2+1 / p<0$ and $p>(n-1) /(n+s-1)$, from equation (5.1), the first term of equation (5.3) is dominated by

$$
\begin{aligned}
& \int_{|x| \geq R} \int_{|y| \geq R,|x-y| \leq|x| / 2} \frac{\left|D^{k+1} u(\xi)\right|^{p} d y d x}{|x-y|^{n+p(s+2 \alpha-k-1+1 / p)-p}} \\
& \quad \leq c\|\phi\|_{B_{p}^{s}(\partial \Omega)}^{p} \int_{|x| \geq R} \frac{1}{|x|^{p n-2 p \alpha+(k+1) p}} \\
& \quad \int_{|x-y| \leq|x| / 2} \frac{d y d x}{|x-y|^{n+p(s+2 \alpha-k-2+1 / p)}} \\
& \quad \leq c\|\phi\|_{B_{p}^{s}(\partial \Omega)}^{p} \int_{|x| \geq R} \frac{d x}{|x|^{p(n+s-1)+1}} \\
& \quad=c R^{-p(n+s-1)-1+n}\|\phi\|_{B_{p}^{s}(\partial \Omega)}^{p}
\end{aligned}
$$

Since $|x|,|y| \geq R$, by equation (5.1), the second term of equation (5.3) 
is dominated by

$$
\begin{aligned}
& \int_{|x| \geq R} \int_{|y| \geq R,|x-y| \geq|x| / 2} \frac{\left|D^{k} u(x)\right|^{p}+\left|D^{k} u(y)\right|^{p}}{|x-y|^{n+p(s+2 \alpha-k-1+1 / p)}} d y d x \\
& \leq\|\phi\|_{B_{p}^{s}(\partial \Omega)}^{p} \int_{|x| \geq R} \frac{1}{|x|^{p(n-2 \alpha+k)}} \\
& \int_{|y| \geq R,|x-y| \geq|x| / 2} \frac{d y d x}{|x-y|^{n+p(s+2 \alpha-k-1+1 / p)}} \\
& +\|\phi\|_{B_{p}^{s}(\partial \Omega)}^{p} \int_{|x| \geq R} \\
& \int_{|y| \geq R,|x-y| \leq|x| / 2} \frac{d y d x}{|x-y|^{n+p(s+2 \alpha-k-1+1 / p)}|y|^{p(n-2 \alpha+k)}} .
\end{aligned}
$$

Since $p>(n-1) /(n+s-1)$, the second term on the right-hand side of equation (5.4) is dominated by $R^{-p(n+s-1)-1+n}\|\phi\|_{B_{p}^{s}(\partial \Omega)}^{p}$. Note that

$$
\begin{aligned}
& \int_{|x| \geq R} \int_{R \leq|y| \leq 2|x|} \frac{d y d x}{|x|^{n+p(s+2 \alpha-k-1+1 / p)|y|^{p n-2 p \alpha+k p}}} \\
& \leq c \begin{cases}R^{-p n+2 p \alpha+n} \int_{|x| \geq R} \frac{d x}{|x|^{n+p(s+2 \alpha-k-1+1 / p)}} & (p n-2 p \alpha+k p>n) \\
\int_{|x| \geq R} \frac{\ln |x| d x}{|x|^{n+p(s+2 \alpha-k-1+1 / p)}} & (p n-2 p \alpha+k p=n) \\
\int_{|x| \geq R} \mid \frac{d x}{|x|^{p(n+s-k-1)+1}} & (p n-2 p \alpha+k p<n)\end{cases} \\
& \leq c R^{-p(n-k-1+s)-1+n} \ln R .
\end{aligned}
$$

Then, since $p>(n-1) /(n+s-1)$, the first term on the right-hand side of equation (5.4) is dominated by

$$
\begin{aligned}
\|\phi\|_{B_{p}^{s}(\partial \Omega)}^{p} \times & \int_{|x| \geq R} \int_{|y| \geq R,|x-y| \geq|x| / 2} \frac{d y d x}{|x-y|^{n+p(s+2 \alpha-k-1+1 / p)|y|^{p n-2 p \alpha+k p}}} \\
\leq & c\left(\int_{|x| \geq R} \int_{R \leq|y| \leq 2|x|} \frac{d y d x}{|x|^{n+p(s+2 \alpha-k-1+1 / p)|y|^{p n-2 p \alpha+k p}}}\right. \\
& \left.\quad+\int_{|x| \geq R} \int_{|y| \geq 2|x|} \frac{d y d x}{|y|^{n+p(n+s-1)+1}}\right) \\
\leq & c R^{-p(n-1+s)-1+n} \ln R .
\end{aligned}
$$

Therefore, $A_{1}+A_{2}+A_{3} \leq c_{R}\|\phi\|_{B_{p}^{s}(\partial \Omega)}^{p}$, and hence equation (1.5) follows. 


\section{Proof of Theorem 1.4.}

Theorem 6.1. Let $1-2 \alpha-1 / p<s<0$. For $\phi \in B_{p}^{s}(\partial \Omega)$, let $u=\mathcal{S}_{2 \alpha} \phi$ be the layer potential defined in equation (1.3). Then the Fourier transform of $u$ is

$$
\widehat{u}(\xi)=|\xi|^{-2 \alpha}\left\langle\phi, e^{2 \pi i \xi \cdot}\right\rangle_{\left(B_{p}^{s}(\partial \Omega), B_{p^{\prime}}^{-s}(\partial \Omega)\right)},
$$

and $u$ is a weak solution of

$$
(-\triangle)^{\alpha} u=0 \quad \text { in } \mathbb{R}^{n} \backslash \partial \Omega .
$$

Proof. For the proof of equation (6.1), let $\phi \in C^{2}(\partial \Omega)$ and $\psi \in$ $C_{\mathrm{c}}^{\infty}\left(\mathbb{R}^{n}\right)$. Then,

$$
\begin{aligned}
\int_{\mathbb{R}^{n}} u(x) \psi(x) d x & =c(n, s) \int_{\partial \Omega} \phi(Q) \int_{\mathbb{R}^{n}} \frac{\psi(x) d x}{|x-Q|^{n-2 \alpha}} d Q \\
& =\int_{\partial \Omega} \phi(Q) \int_{\mathbb{R}^{n}}|\xi|^{-2 \alpha} e^{2 \pi i \xi \cdot Q} \widehat{\widehat{\psi}(\xi)} d \xi d Q \\
& =\int_{\mathbb{R}^{n}} \overline{\widehat{\psi}(\xi)}|\xi|^{-2 \alpha} \int_{\partial \Omega} \phi(Q) e^{2 \pi i \xi \cdot Q} d Q d \xi
\end{aligned}
$$

and hence,

$$
\widehat{u}(\xi)=|\xi|^{-2 \alpha} \int_{\partial \Omega} \phi(Q) e^{2 \pi i \xi \cdot Q} d Q .
$$

Since $C^{2}(\partial \Omega)$ is dense in $B_{p}^{s}(\partial \Omega)$, we obtain equation (6.1) for all $\phi \in B_{p}^{s}(\partial \Omega)$.

To prove equation (6.2), suppose that $\phi \in C^{2}(\partial \Omega)$ and $\psi \in C^{\infty}\left(\mathbb{R}^{n} \backslash\right.$ $\partial \Omega)$. Then, from equation (6.1),

$$
\begin{aligned}
\int_{\mathbb{R}^{n}} u(x)(-\triangle)^{\alpha} \psi(x) d x & =\int_{\mathbb{R}^{n}}|\xi|^{2 \alpha} \widehat{u}(\xi) \overline{\widehat{\psi}(\xi)} d \xi \\
& =\int_{\mathbb{R}^{n}} \widehat{\widehat{\psi}(\xi)} \int_{\partial \Omega} e^{-2 \pi i \xi \cdot Q} \phi(Q) d Q d \xi \\
& =\int_{\partial \Omega} \phi(Q) \overline{\int_{\mathbb{R}^{n}} e^{2 \pi i \xi \cdot Q} \widehat{\psi}(\xi) d \xi} d Q \\
& =\int_{\partial \Omega} \phi(Q) \psi(Q) d Q=0 .
\end{aligned}
$$


Since $(-\triangle)^{t}: \dot{B}_{p}^{s}\left(\mathbb{R}^{n}\right) \rightarrow \dot{B}_{p}^{s-2 t}\left(\mathbb{R}^{n}\right)$ is an isomorphism,

$$
\begin{aligned}
& \left|\int_{\mathbb{R}^{n}} u(x)(-\triangle)^{\alpha} \psi(x) d x\right| \\
& \quad=\left|\int_{\mathbb{R}^{n}}(-\triangle)^{(s+2 \alpha-1+1 / p) / 2} u(x)(-\triangle)^{(-s+1-1 / p) / 2} \psi(x) d x\right| \\
& \quad \leq\left\|(-\triangle)^{(s+2 \alpha-1+1 / p) / 2} u\right\|_{\dot{B}_{p}^{0}\left(\mathbb{R}^{n}\right)} \\
& \left\|(-\triangle)^{(-s+1-1 / p) / 2} \psi\right\|_{\dot{B}_{p^{\prime}}^{0}\left(\mathbb{R}^{n}\right)} \\
& \quad \leq\|u\|_{\dot{B}_{p}^{s+2 \alpha-1+1 / p}\left(\mathbb{R}^{n}\right)}\|\psi\|_{\dot{B}_{p^{\prime}}^{-s+1-1 / p}\left(\mathbb{R}^{n}\right)} \\
& \leq c\|\phi\|_{B_{p}^{s}(\partial \Omega)}\|\psi\|_{B_{p^{\prime}}^{-s+1-1 / p}\left(\mathbb{R}^{n}\right)^{\prime}}
\end{aligned}
$$

Let $\phi_{k} \in C^{2}(\partial \Omega)$ be such that $\phi_{k} \rightarrow \phi$ in $B_{p}^{s}(\partial \Omega)$, and put $u_{k}=\mathcal{S} \phi_{k}$. Then,

$$
\left|\int_{\mathbb{R}^{n}}\left(u_{k}(x)-u(x)\right)(-\triangle)^{\alpha} \psi(x) d x\right| \leq c\left\|\phi_{k}-\phi\right\|_{B_{p}^{s}(\partial \Omega)}\|\psi\|_{B_{p^{\prime}}^{-s+1-1 / p}\left(\mathbb{R}^{n}\right)},
$$

which tends to 0 as $k$ tends to infinity. Hence, since $C^{2}(\partial \Omega)$ is a dense subspace of $B_{p}^{s}(\partial \Omega)$, equation (6.3) holds for $\phi \in B_{p}^{s}(\partial \Omega)$, and so we get equation (6.2) for all $\phi \in B_{p}^{s}(\partial \Omega)$.

Proof of Theorem 1.4. Based on Corollary 4.4, $S_{2 \alpha}: B_{p}^{t-2 \alpha+1}(\partial \Omega) \rightarrow$ $B_{p}^{t}(\partial \Omega)$ is bijective for $0<t<1$ and $1<p<\infty$.

To demonstrate the existence of a solution, let $g \in B_{p}^{t}(\partial \Omega)$. Based on the bijectivity of $S_{2 \alpha}: B_{p}^{t-2 \alpha+1}(\partial \Omega) \rightarrow B_{p}^{t}(\partial \Omega)$, there is a $\phi \in$ $B_{p}^{t-2 \alpha+1}(\partial \Omega)$ such that $S_{2 \alpha} \phi=g$. Let $u=\mathcal{S}_{2 \alpha} \phi$, defined by equation (1.3). Then, from Theorem $6.1, u$ is a weak solution of equation (6.2), and from Theorem 1.2, $u$ satisfies equation (1.7). Hence, the proof of Theorem 1.4 is now complete.

\section{Appendix.}

7.1. Proof of equation (4.4). Because the proofs of the two inequalities in equation (4.4) are similar, we only prove the first. Let

$$
H_{k} f\left(x^{\prime}\right):=\int_{\mathbb{R}^{n-1}} L_{k}\left(x^{\prime}, y^{\prime}\right) f\left(y^{\prime}\right) d y^{\prime}, \quad k=1,2,3 .
$$


Here, $L_{k}\left(x^{\prime}, y^{\prime}\right):=\eta_{i}\left(x^{\prime}, \Psi\left(x^{\prime}\right)\right) \lambda_{i}\left(y^{\prime}, \Psi\left(y^{\prime}\right)\right) K_{k}\left(x^{\prime}, y^{\prime}\right)$, where

$$
\begin{aligned}
K_{1}\left(x^{\prime}, y^{\prime}\right):= & \int_{B^{\prime}(7 \epsilon)} A\left(z^{\prime}\right) \Gamma_{2 \alpha}\left(x^{\prime}-z^{\prime}, \Psi\left(x^{\prime}\right)-\Psi\left(z^{\prime}\right)\right) \\
& \Gamma_{3-2 \alpha}\left(y^{\prime}-z^{\prime}, \Psi\left(y^{\prime}\right)-\Psi\left(z^{\prime}\right)\right) d z^{\prime} \\
K_{2}\left(x^{\prime}, y^{\prime}\right):= & \int_{B^{\prime}(7 \epsilon)} \kappa_{i}\left(z^{\prime}, 0\right) \Gamma_{3-2 \alpha}\left(y^{\prime}-z^{\prime}, \Psi\left(y^{\prime}\right)-\Psi\left(z^{\prime}\right)\right) \\
& \left(\Gamma_{2 \alpha}\left(x^{\prime}-z^{\prime}, \Psi\left(x^{\prime}\right)-\Psi\left(z^{\prime}\right)\right)-\Gamma_{2 \alpha}\left(x^{\prime}-z^{\prime}, 0\right)\right) d z^{\prime} \\
K_{3}\left(x^{\prime}, y^{\prime}\right):= & \int_{B^{\prime}(7 \epsilon)} \kappa_{i}\left(z^{\prime}, 0\right) \Gamma_{2 \alpha}\left(x^{\prime}-z^{\prime}, 0\right) \\
& \left(\Gamma_{3-2 \alpha}\left(y^{\prime}-z^{\prime}, \Psi\left(y^{\prime}\right)-\Psi\left(z^{\prime}\right)\right)-\Gamma_{3-2 \alpha}\left(y^{\prime}-z^{\prime}, 0\right)\right) d z^{\prime},
\end{aligned}
$$

with $A\left(z^{\prime}\right):=\kappa_{i}\left(z^{\prime}, \Psi\left(z^{\prime}\right)\right) \sqrt{1+\left|\nabla \Psi\left(z^{\prime}\right)\right|^{2}}-\kappa_{i}\left(z^{\prime}, 0\right)$. We also define

$$
\begin{aligned}
H_{4} f\left(x^{\prime}\right):= & \eta_{i}\left(x^{\prime}, \Psi\left(x^{\prime}\right)\right) \lambda_{i}\left(y^{\prime}, \Psi\left(y^{\prime}\right)\right) \int_{B^{\prime}(12 \epsilon)} f\left(y^{\prime}\right) B\left(y^{\prime}\right) \\
& \int_{B^{\prime}(7 \epsilon)} \kappa_{i}\left(z^{\prime}, 0\right) \Gamma_{2 \alpha}\left(x^{\prime}-z^{\prime}, 0\right) \Gamma_{3-2 \alpha}\left(y^{\prime}-z^{\prime}, 0\right) d z^{\prime} d y^{\prime},
\end{aligned}
$$

where $B\left(z^{\prime}\right):=\lambda_{i}\left(z^{\prime}, \Psi\left(z^{\prime}\right)\right) \sqrt{1+\left|\nabla \Psi\left(z^{\prime}\right)\right|^{2}}-\lambda_{i}\left(z^{\prime}, 0\right)$. From the definitions of $\kappa_{i}, \lambda_{i}$ and $\Psi$, we have

$$
\begin{aligned}
\left|A\left(z^{\prime}\right)\right| & \leq c \epsilon, \quad\left|B\left(z^{\prime}\right)\right| \leq c \epsilon, \\
\left|D_{z^{\prime}} A\left(z^{\prime}\right)\right| & \leq c, \quad\left|D_{z^{\prime}} B\left(z^{\prime}\right)\right| \leq c .
\end{aligned}
$$

Note that $I_{11}^{i} \phi-I_{111}^{i} \phi=H_{1} \phi+H_{2} \phi+H_{3} \phi+H_{4} \phi$.

First, we estimate $\left\|H_{1} f\right\|_{L^{p}\left(B^{\prime}(2 \epsilon)\right)}$ based on the direct calculation

$$
\begin{aligned}
\left.\left|L_{1}\left(x^{\prime}, y^{\prime}\right)\right| \leq c \epsilon \int_{B^{\prime}(7 \epsilon)} \Gamma_{2 \alpha}\left(x^{\prime}-z^{\prime}, 0\right)\right) \Gamma_{3-2 \alpha}\left(y^{\prime}-z^{\prime}, 0\right) d z^{\prime} & \chi_{B^{\prime}(2 \epsilon)}\left(x^{\prime}\right) \chi_{B^{\prime}(12 \epsilon)}\left(y^{\prime}\right) \\
\leq & c \epsilon \frac{\chi_{B^{\prime}(2 \epsilon)}\left(x^{\prime}\right) \chi_{B^{\prime}(12 \epsilon)}\left(y^{\prime}\right)}{\left|x^{\prime}-y^{\prime}\right|^{n-2}}
\end{aligned}
$$


where $\chi_{S}$ denotes the characteristic function of the set $S$. Let

$$
\frac{1}{q}-\frac{1}{p}<\frac{2 n-3}{n-1} \quad \text { and } \quad \frac{1}{r}=1+\frac{1}{p}-\frac{1}{q} .
$$

Then,

$$
\begin{array}{r}
\left|L_{1}\left(x^{\prime}, y^{\prime}\right) f\left(y^{\prime}\right)\right|=\left|L_{1}\left(x^{\prime}, y^{\prime}\right)\right|^{r(1-1 / q)}\left|L_{1}\left(x^{\prime}, y^{\prime}\right)\right|^{r / p} \\
\left|f\left(y^{\prime}\right)\right|^{p / q}\left|f\left(y^{\prime}\right)\right|^{q(1 / q-1 / p)} .
\end{array}
$$

Using the Hölder inequality, from equation (7.2), for $x^{\prime} \in B^{\prime}(0,2 \epsilon)$,

$$
\begin{gathered}
\left|\int_{\mathbb{R}^{n-1}} L_{1}\left(x^{\prime}, y^{\prime}\right) f\left(y^{\prime}\right) d y^{\prime}\right| \leq\left(\int_{B^{\prime}(0,12 \epsilon)}\left|L_{1}\left(x^{\prime}, y^{\prime}\right)\right|^{r} d y^{\prime}\right)^{1-1 / q} \\
\left(\int_{B^{\prime}(0,12 \epsilon)}\left|L_{1}\left(x^{\prime}, y^{\prime}\right)\right|^{r}\left|f\left(y^{\prime}\right)\right|^{q} d y^{\prime}\right)^{1 / p} \\
\left(\int_{B^{\prime}(0,12 \epsilon)}\left|f\left(y^{\prime}\right)\right|^{q} d y^{\prime}\right)^{1 / q-1 / p} \\
\leq c \epsilon^{((n-1)-(n-3) r)(1-1 / q)}\|f\|_{L^{q}\left(B^{\prime}(0,12 \epsilon)\right)}^{1-q / p} \\
\left(\int_{B^{\prime}(0,12 \epsilon)}\left|L_{1}\left(x^{\prime}, y^{\prime}\right)\right|^{r}\left|f\left(y^{\prime}\right)\right|^{q} d y^{\prime}\right)^{1 / p} .
\end{gathered}
$$

Hence,

$$
\begin{gathered}
\left\|H_{1} f\right\|_{L^{p}\left(B^{\prime}\left(\mathbb{R}^{n-1}\right)\right)} \\
=\left(\int_{B^{\prime}(0,2 \epsilon)}\left|\int_{B^{\prime}(0,12 \epsilon)} L_{1}\left(x^{\prime}, y^{\prime}\right) f\left(y^{\prime}\right) d y^{\prime}\right|^{p} d x^{\prime}\right)^{1 / p} \\
\leq c \epsilon^{((n-1)-(n-3) r)(1-1 / q)} \\
\epsilon^{((n-1)-(n-3) r) / p}\|f\|_{L^{q}\left(B^{\prime}(0,12 \epsilon)\right)}^{1-q / p} \\
\left(\int_{B^{\prime}(0,12 \epsilon)}\left|f\left(y^{\prime}\right)\right|^{q} d y^{\prime}\right)^{1 / p}=c \epsilon^{((n-1)-(n-3) r) / r}\|f\|_{L^{q}\left(B^{\prime}(0,12 \epsilon)\right)} \\
\leq c \epsilon^{((n-1)-(n-3) r) / r} \epsilon^{(n-1)\left(q^{-1}-p^{-1}\right)}\|f\|_{L^{p}\left(B^{\prime}(0,12 \epsilon)\right)} \\
=c \epsilon^{2}\|f\|_{L^{p}\left(B^{\prime}(0,12 \epsilon)\right)}
\end{gathered}
$$


Next, we estimate $\left\|D H_{1} f\right\|_{L^{p}\left(\mathbb{R}^{n-1}\right)}$. Note that

$$
\begin{array}{r}
D_{x^{\prime}} \Gamma_{2 \alpha}\left(x^{\prime}-z^{\prime}, \Psi\left(x^{\prime}\right)-\Psi\left(z^{\prime}\right)\right)=-D_{z^{\prime}} \Gamma_{2 \alpha}\left(x^{\prime}-z^{\prime}, \Psi\left(x^{\prime}\right)-\Psi\left(z^{\prime}\right)\right) \\
+D_{n} \Gamma_{2 \alpha}\left(x^{\prime}-z^{\prime}, \Psi\left(x^{\prime}\right)-\Psi\left(z^{\prime}\right)\right)\left(D \Psi\left(x^{\prime}\right)-D \Psi\left(z^{\prime}\right)\right),
\end{array}
$$

and

$$
\begin{gathered}
D_{y^{\prime}} \Gamma_{3-2 \alpha}\left(y^{\prime}-z^{\prime}, \Psi\left(y^{\prime}\right)-\Psi\left(z^{\prime}\right)\right)=-D_{z^{\prime}} \Gamma_{3-2 \alpha}\left(y^{\prime}-z^{\prime}, \Psi\left(y^{\prime}\right)-\Psi\left(z^{\prime}\right)\right) \\
+D_{n} \Gamma_{3-2 \alpha}\left(y^{\prime}-z^{\prime}, \Psi\left(y^{\prime}\right)-\Psi\left(z^{\prime}\right)\right)\left(D \Psi\left(y^{\prime}\right)-D \Psi\left(z^{\prime}\right)\right) .
\end{gathered}
$$

Hence, using the integration by parts, we have

$$
D_{x^{\prime}} L_{1}\left(x^{\prime}, y^{\prime}\right)=-D_{y^{\prime}} L_{1}\left(x^{\prime}, y^{\prime}\right)+G_{1}\left(x^{\prime}, y^{\prime}\right),
$$

where

$$
\begin{aligned}
G_{1}\left(x^{\prime}, y^{\prime}\right):= & D_{x^{\prime}} \eta_{i}\left(x^{\prime}\right) \lambda_{i}\left(y^{\prime}\right) K_{1}\left(x^{\prime}, y^{\prime}\right) \\
- & \eta_{i}\left(x^{\prime}\right) D_{y^{\prime}} \lambda_{i}\left(y^{\prime}\right) K_{1}\left(x^{\prime}, y^{\prime}\right) \\
+ & \eta_{i}\left(x^{\prime}\right) \lambda_{i}\left(y^{\prime}\right) \int_{B^{\prime}(7 \epsilon)} D_{z^{\prime}} A\left(z^{\prime}\right) \Gamma_{2 \alpha}\left(x^{\prime}-z^{\prime}, \Psi\left(x^{\prime}\right)-\Psi\left(z^{\prime}\right)\right) \\
& \Gamma_{3-2 \alpha}\left(y^{\prime}-z^{\prime}, \Psi\left(y^{\prime}\right)-\Psi\left(z^{\prime}\right)\right) d z^{\prime} \\
+ & \eta_{i}\left(x^{\prime}\right) \lambda_{i}\left(y^{\prime}\right) \int_{B^{\prime}(7 \epsilon)} A\left(z^{\prime}\right) D_{n} \Gamma_{2 \alpha}\left(x^{\prime}-z^{\prime}, \Psi\left(x^{\prime}\right)-\Psi\left(z^{\prime}\right)\right) \\
& \left(D \Psi\left(x^{\prime}\right)-D \Psi\left(z^{\prime}\right)\right) \Gamma_{3-2 \alpha}\left(x^{\prime}-z^{\prime}, \Psi\left(x^{\prime}\right)-\Psi\left(z^{\prime}\right)\right) d z^{\prime} \\
+ & \eta_{i}\left(x^{\prime}\right) \lambda_{i}\left(y^{\prime}\right) \int_{B^{\prime}(7 \epsilon)} A\left(z^{\prime}\right) \Gamma_{2 \alpha}\left(x^{\prime}-z^{\prime}, \Psi\left(x^{\prime}\right)-\Psi\left(z^{\prime}\right)\right) \\
& D_{n} \Gamma_{3-2 \alpha}\left(x^{\prime}-z^{\prime}, \Psi\left(x^{\prime}\right)-\Psi\left(z^{\prime}\right)\right)\left(D \Psi\left(x^{\prime}\right)-D \Psi\left(z^{\prime}\right)\right) d z^{\prime} .
\end{aligned}
$$

Note that

$$
\begin{aligned}
\left|D_{n} \Gamma_{2 \alpha}\left(x^{\prime}-z^{\prime}, \Psi\left(x^{\prime}\right)-\Psi\left(z^{\prime}\right)\right)\left(D \Psi\left(x^{\prime}\right)-D \Psi\left(z^{\prime}\right)\right)\right| & \\
\leq & \leq \frac{c}{\left|x^{\prime}-z^{\prime}\right|^{n-2 \alpha}}
\end{aligned}
$$

and

$$
\begin{aligned}
\left|D_{n} \Gamma_{3-2 \alpha}\left(y^{\prime}-z^{\prime}, \Psi\left(y^{\prime}\right)-\Psi\left(z^{\prime}\right)\right)\left(D \Psi\left(y^{\prime}\right)-D \Psi\left(z^{\prime}\right)\right)\right| & \\
& \leq \frac{c}{\left|y^{\prime}-z^{\prime}\right|^{n-3+2 \alpha}} .
\end{aligned}
$$


Hence, using equation (7.1),

$$
\begin{aligned}
\left|G_{1}\left(x^{\prime}, y^{\prime}\right)\right| \leq & c\left(\int_{B^{\prime}(7 \epsilon)} \Gamma_{2 \alpha}\left(x^{\prime}-z^{\prime}, 0\right) \Gamma_{3-2 \alpha}\left(y^{\prime}-z^{\prime}, 0\right) d z^{\prime}\right. \\
& \left.+\epsilon \int_{B^{\prime}(7 \epsilon)} \Gamma_{2 \alpha}\left(x^{\prime}-z^{\prime}, 0\right) \Gamma_{3-2 \alpha}\left(x^{\prime}-z^{\prime}, 0\right) d z^{\prime}\right) \\
\leq & \chi_{B^{\prime}(2 \epsilon)}\left(x^{\prime}\right) \chi_{B^{\prime}(12 \epsilon)}\left(y^{\prime}\right)
\end{aligned}
$$

and, with the same calculation to equation (7.3),

$$
\left\|H_{12} f\right\|_{L^{p}\left(B^{\prime}(2 \epsilon)\right)} \leq c \epsilon\|f\|_{L^{p}\left(B^{\prime}(12 \epsilon)\right)},
$$

where $H_{12} f\left(x^{\prime}\right)=\int_{\mathbb{R}^{n-1}} G_{1}\left(x^{\prime}, y^{\prime}\right) f\left(y^{\prime}\right) d y^{\prime}$.

Let

$$
H_{13} f\left(x^{\prime}\right)=\int_{\mathbb{R}^{n-1}} D_{y^{\prime}} L_{1}\left(x^{\prime}, y^{\prime}\right) f\left(y^{\prime}\right) d y^{\prime} .
$$

To show the $L^{2}$-boundedness of $H_{13}$, we use the following proposition [20, Theorem 7.3].

Proposition 7.1. Let $T$ be a singular integral with kernel $L$, that is,

$$
T f\left(x^{\prime}\right)=\int_{\mathbb{R}^{n-1}} L\left(x^{\prime}, y^{\prime}\right) f\left(y^{\prime}\right) d y^{\prime}, \quad x^{\prime} \notin \operatorname{supp} f,
$$

for $f \in \mathcal{S}$. Suppose that, for $0<\gamma \leq 1$, the kernel $L$ satisfies

$$
\begin{aligned}
& \left|L\left(x^{\prime}, y^{\prime}\right)\right| \leq A\left|x^{\prime}-y^{\prime}\right|^{-n+1}, \\
& \left|L\left(x^{\prime}, y^{\prime}\right)-L\left(x_{0}^{\prime}, y^{\prime}\right)\right| \leq A \frac{\left|x^{\prime}-x_{0}^{\prime}\right|^{\gamma}}{\left|x^{\prime}-y^{\prime}\right|^{n-1+\gamma}} \quad \text { if }\left|x^{\prime}-x_{0}\right| \leq \frac{\left|x^{\prime}-y^{\prime}\right|}{2}, \\
& \left|L\left(x^{\prime}, y^{\prime}\right)-L\left(x^{\prime}, y_{0}^{\prime}\right)\right| \leq A \frac{\left|y^{\prime}-y_{0}^{\prime}\right|^{\gamma}}{\left|x^{\prime}-y^{\prime}\right|^{n-1+\gamma}} \quad \text { if }\left|y^{\prime}-y_{0}^{\prime}\right| \leq \frac{\left|x^{\prime}-y^{\prime}\right|}{2} .
\end{aligned}
$$

Then, $T$ extends to a bounded linear operator from $L^{2}\left(\mathbb{R}^{n-1}\right)$ to itself if and only if both $T$ and $T^{*}$ are restrictedly bounded, in the sense that

$$
\left\|T \phi^{R, x_{0}^{\prime}}\right\|_{L^{2}\left(\mathbb{R}^{n-1}\right)} \leq A R^{(n-1) / 2}
$$

and

$$
\left\|T^{*} \phi^{R, x_{0}^{\prime}}\right\|_{L^{2}\left(\mathbb{R}^{n-1}\right)} \leq A R^{(n-1) / 2}
$$


for all $x_{0} \in \mathbb{R}^{n-1}$ and $R>0$, where $\phi^{R, x_{0}^{\prime}}\left(x^{\prime}\right):=\phi\left(\left(x^{\prime}-x_{0}^{\prime}\right) / R\right)$ and $\phi$ is a bump function, that is, $\phi \in C_{\mathrm{c}}^{\infty}\left(B^{\prime}(1)\right)$ such that $\left|D \phi\left(x^{\prime}\right)\right| \leq 1$. In this case,

$$
\|T\|_{L^{2} \rightarrow L^{2}} \leq c A
$$

Now we will show that $D_{y^{\prime}} L_{1}\left(x^{\prime}, y^{\prime}\right)$ satisfies the conditions of Proposition 7.1. From equation (7.1), we have

$$
\begin{aligned}
&\left|D_{y^{\prime}} L_{1}\left(x^{\prime}, y^{\prime}\right)\right| \leq c \epsilon \chi_{B^{\prime}(2 \epsilon)}\left(x^{\prime}\right) \chi_{B^{\prime}(12 \epsilon)}\left(y^{\prime}\right) \int_{B^{\prime}(7 \epsilon)} \frac{d z^{\prime}}{\left|x^{\prime}-z^{\prime}\right|^{n-2 \alpha}\left|x^{\prime}-z^{\prime}\right|^{n-2+2 \alpha}} \\
& \leq c \epsilon\left|x^{\prime}-y^{\prime}\right|^{-n+1} \chi_{B^{\prime}(2 \epsilon)}\left(x^{\prime}\right) \chi_{B^{\prime}(12 \epsilon)}\left(y^{\prime}\right) .
\end{aligned}
$$

For $\left|x^{\prime}-x_{0}^{\prime}\right| \leq\left|x^{\prime}-y^{\prime}\right| / 2$, based on the mean-value theorem, there exists a $\xi^{\prime}$ between $x^{\prime}$ and $x_{0}^{\prime}$, such that

$$
\begin{aligned}
& \left|D_{y^{\prime}} L_{1}\left(x_{0}^{\prime}, y^{\prime}\right)-D_{y^{\prime}} L_{1}\left(x_{0}^{\prime}, y^{\prime}\right)\right| \\
& =\left|D_{x^{\prime}} D_{y^{\prime}} L_{1}\left(\xi^{\prime}, y^{\prime}\right) \cdot\left(x^{\prime}-x_{0}^{\prime}\right)\right| \\
& \leq c\left|D_{x^{\prime}} D_{y^{\prime}} K_{1}\left(x^{\prime}, y^{\prime}\right) \cdot\left(x^{\prime}-x_{0}^{\prime}\right)\right| \\
& \leq c \epsilon \frac{\left|x^{\prime}-x_{0}^{\prime}\right|}{\left|x^{\prime}-y^{\prime}\right|^{n}} \chi_{B^{\prime}(2 \epsilon)}\left(x^{\prime}\right) \chi_{B^{\prime}(12 \epsilon)}\left(y^{\prime}\right),
\end{aligned}
$$

and, for $\left|y^{\prime}-y_{0}^{\prime}\right| \geq\left|x^{\prime}-y^{\prime}\right| / 2$, we have

$$
\begin{aligned}
& \left|D_{y^{\prime}} L_{1}\left(x^{\prime}, y_{0}^{\prime}\right)-D_{y^{\prime}} L_{1}\left(x^{\prime}, y_{0}^{\prime}\right)\right| \\
& =\left|D_{y^{\prime}} D_{y^{\prime}} L_{1}\left(x^{\prime}, \xi^{\prime}\right) \cdot\left(y^{\prime}-y_{0}^{\prime}\right)\right| \\
& \leq c\left|D_{x^{\prime}} D_{y^{\prime}} L_{1}\left(x^{\prime}, y^{\prime}\right) \cdot\left(y^{\prime}-y_{0}^{\prime}\right)\right| \\
& \leq c \epsilon \frac{\left|y^{\prime}-y_{0}^{\prime}\right|}{\left|x^{\prime}-y^{\prime}\right|^{n}} \chi_{B^{\prime}(2 \epsilon)}\left(x^{\prime}\right) \chi_{B^{\prime}(12 \epsilon)}\left(y^{\prime}\right) .
\end{aligned}
$$

Hence, $D_{y^{\prime}} L_{1}$ satisfies the conditions of equation (7.5).

Next, we show that $H_{12}$ satisfies equation (7.6). If $\left|x^{\prime}-x_{0}^{\prime}\right| \geq 2 R$, then

$$
\left|\int_{\mathbb{R}^{n-1}} D_{y^{\prime}} L_{1}\left(x^{\prime}, y^{\prime}\right) \phi^{R, x_{0}^{\prime}}\left(y^{\prime}\right) d y^{\prime}\right| \leq c \epsilon\left|x_{0}^{\prime}-x^{\prime}\right|^{-n+1} R^{n-1} .
$$


For $\left|x^{\prime}-x_{0}^{\prime}\right| \leq 2 R$, we have

$$
\begin{aligned}
\left|\int_{\mathbb{R}^{n-1}} D_{y^{\prime}} L_{1}\left(x^{\prime}, y^{\prime}\right) \phi^{R, x_{0}^{\prime}}\left(y^{\prime}\right) d y^{\prime}\right| & =\left|\int_{\mathbb{R}^{n-1}} L_{1}\left(x^{\prime}, y^{\prime}\right) D \phi^{R, x_{0}^{\prime}}\left(y^{\prime}\right) d y^{\prime}\right| \\
& \leq c \in R^{-1} \int_{\substack{\left|x_{0}^{\prime}-y^{\prime}\right| \leq R \\
\left|x^{\prime}-y^{\prime}\right| \leq 1}} \frac{d y^{\prime}}{\left|x^{\prime}-y^{\prime}\right|^{n-2}} .
\end{aligned}
$$

If $R>1$, then the right-hand side is bounded by

$$
\begin{aligned}
c \epsilon R^{-1} \int_{\substack{\left|x_{0}^{\prime}-y^{\prime}\right| \leq R \\
\left|x^{\prime}-y^{\prime}\right| \leq 1}} \frac{d y^{\prime}}{\left|x^{\prime}-y^{\prime}\right|^{n-2}} & \leq c \epsilon R^{-1} \int_{\left|x^{\prime}-y^{\prime}\right| \leq 1} \frac{d y^{\prime}}{\left|x^{\prime}-y^{\prime}\right|^{n-2}} \\
& =c \epsilon R^{-1} \int_{0}^{1} d t \leq c \epsilon,
\end{aligned}
$$

and, if $R<1$, by

$$
c \epsilon R^{-1} \int_{\left|x^{\prime}-y^{\prime}\right| \leq 2 R} \frac{d y^{\prime}}{\left|x^{\prime}-y^{\prime}\right|^{n-2}} \leq c \epsilon .
$$

Thus,

$$
\begin{aligned}
\int_{\left|x_{0}^{\prime}-x^{\prime}\right| \geq 2 R}\left|H_{13} \phi^{x_{0}^{\prime}, R}\left(x^{\prime}\right)\right|^{2} d x^{\prime} & \leq c \epsilon^{2} R^{2 n-2} \int_{\left|x_{0}^{\prime}-x^{\prime}\right| \geq 2 R} \frac{d x^{\prime}}{\left|x_{0}^{\prime}-x^{\prime}\right|^{2 n-2}} \\
& \leq c \epsilon^{2} R^{n-1},
\end{aligned}
$$

and

$$
\int_{\left|x_{0}^{\prime}-x^{\prime}\right| \leq 2 R}\left|H_{13} \phi^{x_{0}^{\prime}, R}\left(x^{\prime}\right)\right|^{2} d x^{\prime} \leq c \epsilon^{2} \int_{\left|x_{0}^{\prime}-x^{\prime}\right| \leq 2 R} d x^{\prime} \leq c \epsilon^{2} R^{n-1} .
$$

Hence,

$$
\begin{aligned}
\left\|H_{13} \phi^{x_{0}^{\prime}, R}\right\|_{L^{2}\left(\mathbb{R}^{n-1}\right)} \leq & \left(\int_{\left|x_{0}^{\prime}-x^{\prime}\right| \leq 2 R}\left|H_{13} \phi^{x_{0}^{\prime}, R}\left(x^{\prime}\right)\right|^{2} d x^{\prime}\right)^{1 / 2} \\
& +\left(\int_{\left|x_{0}^{\prime}-x^{\prime}\right| \geq 2 R}\left|H_{13} \phi^{x_{0}^{\prime}, R}\left(x^{\prime}\right)\right|^{2} d x^{\prime}\right)^{1 / 2} \\
\leq & c \epsilon R^{(n-1) / 2} .
\end{aligned}
$$

Since the kernel of $H_{13}^{*}$ is $D_{x^{\prime}} L_{1}\left(y^{\prime}, x^{\prime}\right)$, by the same estimate, we obtain

$$
\left\|H_{13}^{*} \phi^{x_{0}^{\prime}, R}\right\|_{L^{2}\left(\mathbb{R}^{n-1}\right)} \leq c \epsilon R^{(n-1) / 2} .
$$


Hence,

$$
\left\|H_{13}\right\|_{L^{2} \rightarrow L^{2}} \leq c \epsilon \quad \text { and } \quad\left\|H_{13}^{*}\right\|_{L^{2} \rightarrow L^{2}} \leq c \epsilon
$$

Let $a$ be an atom, that is, supp $a \subset B^{\prime}\left(x_{0}^{\prime}, r\right),\left|a\left(x^{\prime}\right)\right| \leq r^{-n+1}$ and $\int_{\mathbb{R}^{n-1}} a\left(x^{\prime}\right) d x^{\prime}=0$. Then,

$$
\begin{aligned}
\int_{B^{\prime}\left(x_{0}^{\prime}, 2 r\right)}\left|H_{13} a\left(x^{\prime}\right)\right| d x^{\prime} & \leq(2 r)^{(n-1) / 2}\left(\int_{B^{\prime}\left(x_{0}^{\prime}, 2 r\right)}\left|H_{13} a\left(x^{\prime}\right)\right|^{2} d x^{\prime}\right)^{1 / 2} \\
& \leq c \epsilon r^{(n-1) / 2}\left(\int_{B^{\prime}\left(x_{0}^{\prime}, r\right)}\left|a\left(x^{\prime}\right)\right|^{2} d x^{\prime}\right)^{1 / 2} \leq c \epsilon .
\end{aligned}
$$

Since $\int_{\mathbb{R}^{n-1}} a\left(x^{\prime}\right) d x^{\prime}=0$, for $\left|x_{0}^{\prime}-x^{\prime}\right| \geq 2 r$,

$$
\begin{aligned}
H_{13} a\left(x^{\prime}\right) & =\int_{B^{\prime}\left(x_{0}^{\prime}, 2 r\right)}\left(L_{1}\left(x^{\prime}, y^{\prime}\right)-L_{1}\left(x_{0}^{\prime}, y^{\prime}\right)\right) a\left(y^{\prime}\right) d y^{\prime} \\
& \leq c \epsilon r^{-n+1} \int_{B^{\prime}\left(x_{0}^{\prime}, 2 r\right)} \frac{\left|x^{\prime}-x_{0}^{\prime}\right|}{\left|x^{\prime}-y^{\prime}\right|^{n}} d y^{\prime} \\
& \leq \frac{c \epsilon r}{\left|x^{\prime}-x_{0}^{\prime}\right|^{n}}
\end{aligned}
$$

and hence,

$$
\int_{\left|x^{\prime}-x_{0}^{\prime}\right| \geq 2 r}\left|H_{13} a\left(x^{\prime}\right)\right| d x^{\prime} \leq c \epsilon r \int_{\left|x^{\prime}-x_{0}^{\prime}\right| \geq 2 r} \frac{d x^{\prime}}{\left|x^{\prime}-x_{0}^{\prime}\right|^{n}} \leq c \epsilon .
$$

Therefore,

$$
\int_{\mathbb{R}^{n-1}}\left|H_{13} a\left(x^{\prime}\right)\right| d x^{\prime} \leq c \epsilon,
$$

implying that $\left\|H_{13}\right\|_{H^{1} \rightarrow L^{1}} \leq c \epsilon$, where $H^{1}$ is a Hardy space. For the same reason, $\left\|H_{13}^{*}\right\|_{H^{1} \rightarrow L^{1}} \leq c \epsilon$, so

$$
\left\|H_{13}\right\|_{L^{p} \rightarrow L^{p}} \leq c \epsilon \text { for } 1<p<\infty .
$$

Since $D H_{1} f=H_{12} f+H_{13} f$, based on equations (7.4) and (7.7),

$$
\left\|D H_{1}\right\|_{L^{p}\left(\mathbb{R}^{n-1}\right) \longrightarrow L^{p}\left(\mathbb{R}^{n-1}\right)} \leq c \epsilon .
$$

Hence, $\left\|H_{1}\right\|_{L^{p}\left(\mathbb{R}^{n-1}\right) \rightarrow H_{p}^{1}\left(\mathbb{R}^{n-1}\right)} \leq c \epsilon$.

Next, we estimate $\left\|H_{2} f\right\|_{L^{p} \rightarrow H_{p}^{1}}$. Note that

$$
\left|L_{2}\left(x^{\prime}, y^{\prime}\right)\right| \leq c \epsilon^{2}\left|x^{\prime}-y^{\prime}\right|^{-n+2} \chi_{B^{\prime}(2 \epsilon)}\left(x^{\prime}\right) \chi_{B^{\prime}(12 \epsilon)}\left(y^{\prime}\right)
$$


and $D_{x^{\prime}} K_{2}\left(x^{\prime}, y^{\prime}\right)=-D_{y^{\prime}} L_{2}\left(x^{\prime}, y^{\prime}\right)+G_{2}\left(x^{\prime}, y^{\prime}\right)$, where

$$
\begin{aligned}
G_{2}\left(x^{\prime}, y^{\prime}\right):= & \int_{B^{\prime}(7 \epsilon)} D_{z^{\prime}} \kappa_{i}\left(z^{\prime}, 0\right) \Gamma_{2 \alpha}\left(x^{\prime}-z^{\prime}, \Psi\left(x^{\prime}\right)-\Psi\left(z^{\prime}\right)\right) \\
& \left(\Gamma_{3-2 \alpha}\left(x^{\prime}-z^{\prime}, \Psi\left(x^{\prime}\right)-\Psi\left(z^{\prime}\right)\right)-\Gamma_{3-2 \alpha}\left(y^{\prime}-z^{\prime}, 0\right)\right) d z^{\prime} \\
+ & \int_{B^{\prime}(7 \epsilon)} \kappa_{i}\left(z^{\prime}, 0\right) D_{n} \Gamma_{2 \alpha}\left(x^{\prime}-z^{\prime}, \Psi\left(x^{\prime}\right)-\Psi\left(z^{\prime}\right)\right) \\
& \left(D \Psi\left(x^{\prime}\right)-D \Psi\left(z^{\prime}\right)\right) \Gamma_{3-2 \alpha}\left(x^{\prime}-z^{\prime}, \Psi\left(x^{\prime}\right)-\Psi\left(z^{\prime}\right)\right) d z^{\prime} \\
+ & \int_{B^{\prime}(7 \epsilon)} \kappa_{i}\left(z^{\prime}, 0\right) \Gamma_{2 \alpha}\left(x^{\prime}-z^{\prime}, \Psi\left(x^{\prime}\right)-\Psi\left(z^{\prime}\right)\right) \\
& D_{n} \Gamma_{3-2 \alpha}\left(x^{\prime}-z^{\prime}, \Psi\left(x^{\prime}\right)-\Psi\left(z^{\prime}\right)\right)\left(D \Psi\left(x^{\prime}\right)-D \Psi\left(z^{\prime}\right)\right) d z^{\prime}
\end{aligned}
$$

satisfies

$$
\left|G_{2}\left(x^{\prime}, y^{\prime}\right)\right| \leq c\left|x^{\prime}-y^{\prime}\right|^{n-2} \chi_{B^{\prime}(2 \epsilon)}\left(x^{\prime}\right) \chi_{B^{\prime}(12 \epsilon)}\left(y^{\prime}\right) .
$$

Hence, using the same argument as in the case of $H_{1}$, we can show that

$$
\left\|H_{2}\right\|_{L^{p}\left(B^{\prime}(12 \epsilon)\right) \longrightarrow H_{p}^{1}\left(B^{\prime}(2 \epsilon)\right)} \leq c \epsilon .
$$

Similarly, we have

$$
\left\|H_{3}\right\|_{L^{p}\left(B^{\prime}(12 \epsilon)\right) \longrightarrow H_{p}^{1}\left(B^{\prime}(2 \epsilon)\right)} \leq c \epsilon .
$$

Based on the above arguments,

$$
H_{4}: L^{p}\left(B^{\prime}(12 \epsilon)\right) \longrightarrow H_{p}^{1}\left(B^{\prime}(2 \epsilon)\right)
$$

is a bounded operator. Then, from equation (7.1), we have

$$
\left\|H_{4} \phi\right\|_{H_{p}^{1}\left(B^{\prime}(2 \epsilon)\right)} \leq c\|B \phi\|_{L^{p}\left(B^{\prime}(12 \epsilon)\right)} \leq c \epsilon\|\phi\|_{L^{p}\left(B^{\prime}(12 \epsilon)\right)} .
$$

Hence,

$$
\left\|H_{4}\right\|_{L^{p}\left(B^{\prime}(12 \epsilon)\right) \longrightarrow H_{p}^{1}\left(B^{\prime}(2 \epsilon)\right)} \leq c \epsilon,
$$

and finally,

$$
\begin{aligned}
& \left\|I_{11}-I_{111}\right\|_{L^{p}\left(B^{\prime}(12 \epsilon)\right) H_{p}^{1}\left(B^{\prime}(2 \epsilon)\right)} \\
& \leq\left(\left\|H_{1}\right\|_{L^{p} \rightarrow H_{p}^{1}}+\left\|H_{2}\right\|_{L^{p} \rightarrow H_{p}^{1}}+\left\|H_{3}\right\|_{L^{p} \rightarrow H_{p}^{1}}+\left\|H_{4}\right\|_{L^{p} \rightarrow H_{p}^{1}}\right) \leq c \epsilon .
\end{aligned}
$$




\section{REFERENCES}

1. M.S. Agranovich and B.A. Amosov, Estimates of s-numbers and spectral asymptotics for integral operators of potential type on nonsmooth surfaces, Funk. Anal. Pril. 30 (1996), 1-18 (in Russian); Funct. Anal. Appl. 30 (1996), 75-89 (in English).

2. J. Bergh and J. Löfström, Interpolation spaces, An introduction, SpringerVerlag, Berlin, 1976.

3. K. Bogdan and T. Byczkowski, Potential theory for the $\alpha$-stable Schrödinger operator on bounded Lipschitz domains, Stud. Math. 133 (1999), 53-92.

4. Russell M. Brown, The method of layer potentials for the heat equation in Lipschitz cylinders, Amer. J. Math. 111 (1989), 339-379.

5. , The initial-Neumann problem for the heat equation in Lipschitz cylinders, Trans. Amer. Math. Soc. 320 (1990), 1-52.

6. T.K. Chang, Boundary integral operator for the fractional Laplace equation in a bounded Lipschitz domain, Int. Equat. Operator Theory 72 (2012), 345-361.

7. R.R. Coifman, A. McIntosh and Y. Meyer, L'integrale de Cauchy definit un operateur Borne sur L2 pour les Courbes Lipschitiennes, Ann. Math. 116 (1982), $361-388$.

8. B.E.J. Dahlberg, C.E. Kenig and G.C. Verchota, Boundary value problems for the systems of elastostatics in Lipschitz domains, Duke Math. J. 57 (1988), 109-135.

9. E. Fabes, M. Jodeit and N. Riviere, Potential techniques for boundary value problems on $C^{1}$-domains, Acta Math. 141 (1978), 165-186.

10. E.B. Fabes, C.E. Kenig and G.C. Verchota, The Dirichlet problem for the Stokes system on Lipschitz domains, Duke Math. J. 57 (1988), 769-793.

11. E. Fabes, O. Mendez and M. Mitrea, Boundary layers on Sobolev-Besov spaces and Poisson's equation for the Laplacian in Lipschitz domains, J. Funct. Anal. 159 (1998), 323-368.

12. M. Frazier and B. Jawerth, Decomposition of Besov spaces, Indiana Univ. Math. J. 34 (1985), 777-799.

13. S. Hofmann and J.L. Lewis, $L^{2}$ solvability and representation by caloric layer potentials in time-varying domains, Ann. Math. 144 (1996), 349-420.

14. D. Jerison and C.E. Kenig, The inhomogeneous Dirichlet problem in Lipschitz domains, J. Funct. Anal. 130 (1995), 161-219.

15. A. Jonsson and H. Wallin, A Whitney extension theorem in $L_{p}$ and Besov spaces, Ann. Inst. Fourier 28 (1978), 139-192.

16. M. Mitrea, The method of layer potentials in electromagnetic scattering theory on nonsmooth domains, Duke Math. J. 77 (1995), 111-133.

17. G. Rozenblum and G. Tashchiyan, Eigenvalue asymptotics for potential type operators on Lipschitz surfaces, Russian J. Math. Phys. 13 (2006), 326-339. 
18. Z.W. Shen, Boundary value problems for parabolic Lame systems and a nonstationary linearized system of Navier-Stokes equations in Lipschitz cylinders, Amer. J. Math. 113 (1991), 293-373.

19. E.M. Stein, Singular integrals and differentiability properties of functions, Princeton University Press, Princeton, 1970.

20. Harmonic analysis, real variable methods, orthogonality, and oscillatory integrals, Princeton University Press, Princeton, 1993.

21. G. Verchota, Layer potentials and regularity for the Dirichlet problem for Laplace's equation in Lipschitz domains, J. Funct. Anal. 59 (1984), 572-611.

22. M. Zähle, Harmonic calculus on fractals-A measure geometric approach II, Trans. Amer. Math. Soc. 357 (2005), 3407-3423.

23. _ Riesz potentials and Liouville operators on fractals, Potential Anal. 21 (2004), 193-208.

Department of Mathematics, Yonsei University, 50 Yonsei-ro, SeodaemunGu, Seoul, South Korea 120-749

Email address: chang7357@yonsei.ac.kr 